\title{
Elements in Pointed Invariant Cones in Lie Algebras and Corresponding Affine Pairs
}

\section{Karl-Hermann Neeb ${ }^{1}$ (D) Daniel Oeh ${ }^{1}$}

Received: 18 October 2021 / Revised: 30 November 2021 / Accepted: 2 December 2021 /

Published online: 29 December 2021

(c) The Author(s) 2021

\begin{abstract}
In this note, we study in a finite dimensional Lie algebra $\mathfrak{g}$ the set of all those elements $x$ for which the closed convex hull of the adjoint orbit contains no affine lines; this contains in particular elements whose adjoint orbits generates a pointed convex cone $C_{x}$. Assuming that $\mathfrak{g}$ is admissible, i.e., contains a generating invariant convex subset not containing affine lines, we obtain a natural characterization of such elements, also for non-reductive Lie algebras. Motivated by the concept of standard (Borchers) pairs in QFT, we also study pairs $(x, h)$ of Lie algebra elements satisfying $[h, x]=x$ for which $C_{x}$ pointed. Given $x$, we show that such elements $h$ can be constructed in such a way that ad $h$ defines a 5-grading, and characterize the cases where we even get a 3 -grading.
\end{abstract}

Keywords Lie algebra - Invariant cone - Moment map · Borchers pair - Affine pair . Euler element

Mathematics Subject Classification 22E60 · 22E15

\section{Contents}

1 Introduction . . . . . . . . . . . . . . . . . . . . . . . . . 296

2 Structure of Admissible Lie Algebras . . . . . . . . . . . . . . . . . . . . . . . . . . . . 298

Communicated by Malihe Yousofzadeh.

This research has been supported by DFG-Grant NE 413/10-1.

$\bowtie \quad$ Karl-Hermann Neeb

neeb@math.fau.de

Daniel Oeh

oehd@math.fau.de

1 Department of Mathematics, Friedrich-Alexander-University Erlangen-Nuremberg, Cauerstrasse 11, 91058 Erlangen, Germany 
3 Elements in Pointed Cones . . . . . . . . . . . . . . . . . . . . . . . . . . . . . . 302

3.1 General Observations . . . . . . . . . . . . . . . . . . . . . . . . 302

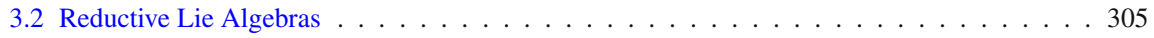

3.3 The Characterization Theorem . . . . . . . . . . . . . . . . . . . . 309

4 Affine Pairs . . . . . . . . . . . . . . . . . . . . . . . . . 316

4.1 Invariance of $W$ Under One-Parameter Groups of Outer Automorphisms . . . . . . . . . . . 317

4.2 Extending Nilpotent Elements to Affine Pairs . . . . . . . . . . . . . . . . . . . . . . . 319

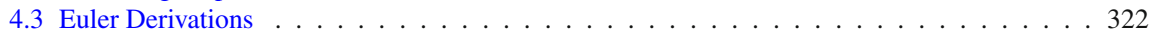

A: Tools Concerning Convexity . . . . . . . . . . . . . . . . . . . . . . . . . 327

B: Tools Concerning Lie Algebras . . . . . . . . . . . . . . . . . . . . . . . . . 328

References . . . . . . . . . . . . . . . . . . . . . . . 329

\section{Introduction}

Convexity properties of adjoint orbits $\mathcal{O}_{x}=\operatorname{Inn}(\mathfrak{g}) x$ in a finite dimensional real Lie algebra, where $\operatorname{Inn}(\mathfrak{g})=\left\langle e^{\operatorname{ad} \mathfrak{g}}\right\rangle$ is the group of inner automorphisms, play a role in many contexts. Most directly, they appear in the theory of invariant convex cones. For instance, if $U: G \rightarrow \mathrm{U}(\mathcal{H})$ is a unitary representation and $\partial U(x)$ denotes the infinitesimal generator of the unitary one-parameter group $(U(\exp t x))_{t \in \mathbb{R}}$, then the positive cone of $U$

$$
C_{U}:=\{x \in \mathfrak{g}:-i \partial U(x) \geq 0\}
$$

is a closed convex invariant cone in $\mathfrak{g}$ which is pointed (contains no affine lines) if and only if $\operatorname{ker}(U)$ is discrete. In the literature pointed generating invariant cones have been studied from the perspective of their interior: If $W \subseteq \mathfrak{g}$ is pointed and generating, then its interior consists of elliptic elements ( $\operatorname{ad} x$ is semisimple with imaginary spectrum) and $W$ is determined by its intersection with a compactly embedded Cartan subalgebra $[5,12]$. Unfortunately, this theory provides not much information on the non-elliptic elements in the boundary of $W$. A notable exception is [6] which, for a simple hermitian Lie algebra, provides a classification of all nilpotent adjoint orbits in an invariant cone.

In the present paper, we address adjoint orbits in invariant cones. For more precise formulations, we introduce some notation. For $x \in \mathfrak{g}$, we write

- $\operatorname{co}(x):=\overline{\operatorname{conv}\left(\mathcal{O}_{x}\right)}$, for the closed convex hull of $\mathcal{O}_{x}$, and

- $C_{x}:=\operatorname{cone}\left(\mathcal{O}_{x}\right)=\overline{\mathbb{R}_{+} \operatorname{conv}\left(\mathcal{O}_{x}\right)}$ for the closed convex cone generated by $x$.

We call a closed convex subset $C$ of a real linear space $V$ pointed if it contains no non-trivial affine lines and generating if $\operatorname{span} C=V$. We study the subsets

$$
\mathfrak{g}_{\mathrm{co}}:=\{x \in \mathfrak{g}: \operatorname{co}(x) \text { pointed }\} \supseteq \mathfrak{g}_{\mathrm{c}}:=\left\{x \in \mathfrak{g}: C_{x} \text { pointed }\right\}
$$

and characterize the elements in this set in terms of explicitly available data.

If $\mathfrak{g}$ is a simple Lie algebra, then we have to distinguish three cases. If $\mathfrak{g}$ is compact, then all sets $\operatorname{co}(x)$ are compact, hence pointed, so that $\mathfrak{g}_{c o}=\mathfrak{g}$. As every convex cone invariant under a compact group contains a fixed point in its interior, we have $\mathfrak{g}_{\mathrm{c}}=\{0\}$ for compact Lie algebras. If $\mathfrak{g}$ is non-compact, then $\mathfrak{g}_{\mathfrak{c}} \neq\{0\}$ is equivalent to $\mathfrak{g}$ being hermitian, i.e., maximal compactly embedded subalgebras have non-trivial center. In 
this case $\mathfrak{g}_{\mathrm{c}}=\mathfrak{g}_{\mathrm{co}}$ is a double cone (cf. Lemma 3.7, Kostant-Vinberg Theorem). If $\mathfrak{g}$ is neither compact nor hermitian, then $\mathfrak{g}_{\mathrm{c}}=\mathfrak{g}_{\mathrm{co}}=\{0\}$.

For a direct sum $\mathfrak{g}=\mathfrak{g}_{1} \oplus \mathfrak{g}_{2}$ we have $\mathfrak{g}_{\text {co }}=\mathfrak{g}_{1, \text { co }} \times \mathfrak{g}_{2, \text { co }}$. This reduces for a reductive Lie algebra the determination of this set to the case of simple hermitian Lie algebras. However, the determination of the subset $\mathfrak{g}_{\mathrm{c}}$ of $\mathfrak{g}_{\mathrm{co}}$ is less obvious for reductive Lie algebras (Proposition 3.14).

This discussion shows that, among the reductive Lie algebras only the quasihermitian ones (all simple ideals are either compact or hermitian), play a role in our context. Beyond reductive Lie algebras, the natural context for our investigation is the class of admissible Lie algebras, i.e., those containing an $\operatorname{Inn}(\mathfrak{g})$-invariant pointed generating closed convex subset $C$ (cf. [12, Def. VII.3.2]). Since every pointed invariant convex subset spans an admissible ideal, we shall assume throughout that $\mathfrak{g}$ is admissible.

It is of vital importance for our arguments, that admissible Lie algebras permit a powerful structure theory. Their coarse structure is given by $\mathfrak{g}=\mathfrak{g}(\mathfrak{l}, V, \mathfrak{z}, \beta)=$ $\mathfrak{z} \oplus V \oplus \mathfrak{l}$, where $\mathfrak{l}$ is a reductive Lie algebra, $V$ an $\mathfrak{l}$-module, $\mathfrak{z}$ a vector space, and $\beta: V \times V \rightarrow \mathfrak{z}$ an l-invariant skew-symmetric bilinear map; the Lie bracket on $\mathfrak{g}$ is given by

$$
\left[(z, v, x),\left(z^{\prime}, v^{\prime}, x^{\prime}\right)\right]=\left(\beta\left(v, v^{\prime}\right), x \cdot v^{\prime}-x^{\prime} \cdot v,\left[x, x^{\prime}\right]\right) \in \mathfrak{z} \oplus V \oplus \mathfrak{l} .
$$

For their fine structure, we use the existence of a compactly embedded Cartan subalgebra $\mathfrak{t}=\mathfrak{z} \oplus \mathfrak{t}_{\mathfrak{l}}$ and the corresponding root decomposition.

The key observation underlying our analysis of the sets $\mathfrak{g}_{\mathrm{c}}$ and $\mathfrak{g}_{\mathrm{co}}$ in Sect.3, is the Reduction Theorem 3.2, asserting that every adjoint orbit in $\mathfrak{g}_{\mathrm{co}}$ intersects the reductive subalgebra $\mathfrak{z}+\mathfrak{l}$. We therefore take in Sect. 3.2 a closer look at the reductive case, where we provide in Propositions 3.10 and 3.14 a complete description of the sets $\mathfrak{g}_{\mathrm{c}}$ and $\mathfrak{g}_{\mathrm{co}}$. The central result in Sect. 3 is the Characterization Theorem 3.20 that characterizes elements $x$ in $\mathfrak{g}_{\mathrm{c}}$ and $\mathfrak{g}_{\mathrm{co}}$ in terms of the closed convex hull $\operatorname{co}_{\mathfrak{z}}(x)$ of the $\mathfrak{z}$-valued Hamiltonian function

$$
H_{x}^{\mathfrak{z}}(v)=p_{\mathfrak{z}}\left(e^{\operatorname{ad} v} x\right)=x_{\mathfrak{z}}+\left[v, x_{V}\right]+\frac{1}{2}\left[v,\left[v, x_{\mathfrak{l}}\right]\right] \quad \text { for } \quad x=x_{\mathfrak{z}}+x_{V}+x_{\mathfrak{l}}, v \in V .
$$

In particular, we show that $\operatorname{co}(x)$ is pointed if and only if $\operatorname{co}_{\mathfrak{z}}(x)$ is pointed and that $C_{x}$ is pointed if, in addition, $\operatorname{co}_{\mathfrak{z}}(x)$ generates a pointed cone whenever the l-component $x_{\mathfrak{l}}$ of $x$ is nilpotent. We also discuss to which extent the pointedness of $C_{x}$ implies the existence of a pointed generating invariant cone $W \subseteq \mathfrak{g}$ containing $x$; which is not always the case (Example 3.25).

In Sect. 4 we study affine pairs $(x, h)$ for a pointed invariant cone $W \subseteq \mathfrak{g}$. These pairs are characterized by the relations

$$
x \in W \quad \text { and } \quad[h, x]=x .
$$

The interest in these pairs stems from their relevance in Algebraic Quantum Field Theory (AQFT), where they arise from unitary representations $(U, \mathcal{H})$ of a corresponding Lie group $G$ and their positive cones $W=C_{U}$ (see (1.1)). If $U$ extends to 
an antiunitary representation of $G \rtimes\left\{\operatorname{id}_{G}, \tau_{G}\right\}$, where $\tau_{G} \in \operatorname{Aut}(G)$ is an involution and the corresponding involution $\tau \in \operatorname{Aut}(\mathfrak{g})$ satisfies $\tau(h)=h$ and $\tau(x)=-x$, then we can associate with $h$ a so-called standard subspace

$$
V:=\operatorname{Fix}\left(U\left(\tau_{G}\right) e^{\pi i \cdot \partial U(h)}\right) \subseteq \mathcal{H}
$$

and these subspaces encode localization data in QFT [2]. In this context $\left(U_{x}, V\right)$ with $U_{x}(t):=U(\exp t x)$ is called a Borchers pair or a standard pair [8], and one would like to understand all those pairs arising from a given unitary representation (see [10] for more details). This leads naturally to the problem to describe and classify affine pairs. For an affine pair $(x, h)$ the element $x$ is nilpotent, hence in particular not elliptic if it is not central. As we know from [11], the most important affine pairs are those for which $h$ in an Euler element of $\mathfrak{g}$, i.e., ad $h$ is diagonalizable with possible eigenvalues $\{-1,0,1\}$ and the Lie algebra $\mathfrak{g}$ is generated by $h$ and the cones $C_{U} \cap \mathfrak{g}_{ \pm 1}(h)$ (see $[15,17]$ for related classification results). Considering representations with discrete kernel then leads to the situation, where the cone $C_{U} \subseteq \mathfrak{g}$ is pointed, so that $\mathfrak{g}=\mathfrak{g}_{U} \rtimes \mathbb{R} h$, and $\mathfrak{g}_{U}=C_{U}-C_{U}$ is an ideal containing the pointed generating invariant cone $C_{U}$.

This motivates our investigations in Sect. 4 , where we start with a nilpotent element $x \in W, W$ a pointed generating invariant cone, and then consider derivations $D$ on $\mathfrak{g}$ satisfying $D x=x$ and $e^{\mathbb{R} D} W=W$. Our first main result on affine pairs is the Existence Theorem 4.7, asserting that, for any nilpotent element $x \in \mathfrak{g}_{\mathrm{co}}$ there exists such a derivation $D$ with $\operatorname{Spec}(D) \subseteq\left\{0, \pm \frac{1}{2}, \pm 1\right\}$. The second main result characterizes the existence of Euler derivations with this property, i.e., where we even have $\operatorname{Spec}(D) \subseteq\{0, \pm 1\}$ (Theorem 4.17).

\section{Notation:}

- For a Lie algebra $\mathfrak{g}$, we write $\operatorname{Inn}(\mathfrak{g})=\left\langle e^{\mathrm{ad}} \mathfrak{g}\right\rangle$ for the group of inner automorphisms. For a Lie subalgebra $\mathfrak{h} \subseteq \mathfrak{g}$, we write $\operatorname{Inn}_{\mathfrak{g}}(\mathfrak{h}) \subseteq \operatorname{Inn}(\mathfrak{g})$ for the subgroup generated by $e^{\operatorname{ad} \mathfrak{h}}$. "Invariance" of subsets of $\mathfrak{g}$ always refers to the group $\operatorname{Inn}(\mathfrak{g})$.

- A subalgebra $\mathfrak{k} \subseteq \mathfrak{g}$ is said to be compactly embedded if the closure of $\operatorname{Inn}_{\mathfrak{g}}(\mathfrak{k})$ in $\operatorname{Aut}(\mathfrak{g})$ is compact. A compactly embedded Cartan subalgebra is a compactly embedded subalgebra which is also maximal abelian.

- For $A \in \operatorname{End}(V)$, we write $V_{\lambda}(A):=\operatorname{ker}\left(A-\lambda \mathrm{id}_{V}\right)$ for the eigenspaces.

- A closed convex cone in a finite dimensional real vector space is simply called a cone. We write cone $(S)=\overline{\mathbb{R}_{+} \operatorname{conv}(S)}$ for the closed convex cone generated by a subset $S$ and

$$
S^{\star}:=\left\{\alpha \in V^{*}:(\forall v \in S) \alpha(v) \geq 0\right\}
$$

for the dual cone.

\section{Structure of Admissible Lie Algebras}

In this section, we collect some relevant results on the structure of admissible Lie algebras. 
Definition 2.1 Let $C$ be a closed convex subset of the finite dimensional real vector space $V$. Then the recession cone of $C$ is

$\lim (C):=\{x \in V: C+x \subseteq C\}=\left\{x \in V: x=\lim _{n \rightarrow \infty} t_{n} c_{n}, c_{n} \in C, t_{n} \rightarrow 0, t_{n} \geq 0\right\}$

(cf. [12, Prop. V.1.6]). The edge of this cone is

$$
H(C):=\lim (C) \cap-\lim (C)=\{x \in V: C+x=C\} .
$$

We say that $C$ is pointed if $H(C)=\{0\}$, which is equivalent to $C$ not containing affine lines ( [12, Prop. V.1.6]).

Definition 2.2 A finite dimensional real Lie algebra $\mathfrak{g}$ is called admissible if it contains an $\operatorname{Inn}(\mathfrak{g})$-invariant pointed generating closed convex subset $C$ (cf. [12, Def. VII.3.2]).

Remark 2.3 If $C \subseteq \mathfrak{g}$ is an invariant pointed closed convex subset, then $\mathfrak{g}_{C}:=$ span $C \unlhd \mathfrak{g}$ is an ideal in which $C$ is also generating, so that $\mathfrak{g}_{C}$ is an admissible Lie algebra.

Definition 2.4 (Spindler construction [18]) Let $\mathfrak{l}$ be a Lie algebra, $V$ an $\mathfrak{l}$-module, $\mathfrak{z}$ a vector space, and $\beta: V \times V \rightarrow \mathfrak{z}$ an l-invariant skew-symmetric bilinear map. Then $\mathfrak{z} \times V \times \mathfrak{l}$ is a Lie algebra with respect to the bracket

$$
\left[(z, v, x),\left(z^{\prime}, v^{\prime}, x^{\prime}\right)\right]=\left(\beta\left(v, v^{\prime}\right), x \cdot v^{\prime}-x^{\prime} \cdot v,\left[x, x^{\prime}\right]\right)
$$

We write $\mathfrak{g}(\mathfrak{l}, V, \mathfrak{z}, \beta)$ for the so-obtained Lie algebra.

The following theorem describes the structure of non-reductive admissible Lie algebras.

Theorem 2.5 Any admissible Lie algebra $\mathfrak{g}$ is of the form $\mathfrak{g}(\mathfrak{l}, V, \mathfrak{z}, \beta)$, where

(a) $\mathfrak{z}=\mathfrak{z}(\mathfrak{g}), \mathfrak{u}=\mathfrak{z}+V$ is the maximal nilpotent ideal.

(b) $\mathfrak{l}$ is reductive and quasihermitian, i.e., all simple ideals are compact or hermitian.

(c) $\mathfrak{l}$ contains a compactly embedded Cartan subalgebra $\mathfrak{t}_{\mathfrak{l}}$, and $\mathfrak{t}:=\mathfrak{z}+\mathfrak{t}_{\mathfrak{l}}$ is a compactly embedded Cartan subalgebra of $\mathfrak{g}$.

(d) $V=[\mathfrak{t}, \mathfrak{u}]=\left[\mathfrak{t}_{\mathfrak{l}}, V\right]$.

(e) There exists an element $f \in \mathfrak{z}^{*}$ such that $(V, f \circ \beta)$ is a symplectic l-module of convex type, i.e., there exists an element $x \in \mathfrak{l}$, such that the Hamiltonian function

$$
H_{x}^{f}: V \rightarrow \mathbb{R}, \quad H_{x}^{f}(v):=f(\beta(x . v, v))
$$

is positive definite.

Conversely, $\mathfrak{g}(\mathfrak{l}, V, \mathfrak{z}, \beta)$ is admissible if (a)-(e) are satisfied. 
Proof Properties (a)-(d) follow from [12, p. 293], combined with [12, Thm. VII.2.26]. Since $\mathfrak{t}$ is compactly embedded, (d) is equivalent to $\mathfrak{z} V\left(\mathfrak{t}_{\mathfrak{l}}\right):=\left\{v \in V:\left[v, \mathfrak{t}_{\mathfrak{l}}\right]=\{0\}\right\}=$ $\{0\}$. Further

$$
\mathfrak{z}_{\mathfrak{z}(\mathfrak{l})}(V):=\{x \in \mathfrak{z}(\mathfrak{l}):[x, V]=\{0\}\}=\mathfrak{z}(\mathfrak{l}) \cap \mathfrak{z}(\mathfrak{g})=\{0\} .
$$

Therefore, [12, Thm. VIII.2.7] implies that, $\mathfrak{g}(\mathfrak{l}, V, \mathfrak{z}, \beta)$ is admissible if (a)-(e) are satisfied.

Corollary 2.6 (Non-degeneracy of $\beta$ ) Let $\mathfrak{g}=\mathfrak{g}(\mathfrak{l}, V, \mathfrak{z}, \beta)$ be admissible and $0 \neq v \in$ $V$. Then there exists an element $w \in V$ with $\beta(v, w)=[v, w] \neq 0$.

Proof In the context of Theorem 2.5(e), we see that $w:=x . v$ satisfies $\beta(x . v, v) \neq 0$.

Lemma 2.7 If $\mathfrak{g}=\mathfrak{g}(\mathfrak{l}, V, \mathfrak{z}, \beta)$ is admissible, then the following assertions hold:

(a) Every abelian ideal of $\mathfrak{g}$ is central.

(b) Every ideal of $\mathfrak{g}$ contained in $V$ is trivial.

Proof (a) Let $\mathfrak{a} \unlhd \mathfrak{g}$ be an abelian ideal and $C \subseteq \mathfrak{g}$ be a pointed generating invariant closed convex subset. For $x \in C$ we then have $e^{\text {ad } \mathfrak{a}} x=x+[\mathfrak{a}, x] \subseteq C$, and, since $C$ is pointed, $[x, \mathfrak{a}]=\{0\}$. As $\mathfrak{g}=\operatorname{span}(C)$, it follows that $[\mathfrak{g}, \mathfrak{a}]=\{0\}$.

(b) If $\mathfrak{a} \subseteq V$ is an ideal of $\mathfrak{g}$, then $[V, \mathfrak{a}] \subseteq[V, V] \cap \mathfrak{a} \subseteq \mathfrak{z} \cap \mathfrak{a}=\{0\}$ implies that $\mathfrak{a}$ is abelian. In view of (a), $\mathfrak{a}$ is central, so that $\mathfrak{a} \subseteq V \cap \mathfrak{z}=\{0\}$.

Definition 2.8 (a) Let $\mathfrak{t} \subseteq \mathfrak{g}$ be a compactly embedded Cartan subalgebra, $\mathfrak{g} \mathbb{C}$ the complexification of $\mathfrak{g}, z=x+i y \mapsto z^{*}:=-x+i y$ the corresponding involution, and $\mathfrak{t}_{\mathbb{C}}$ the corresponding Cartan subalgebra of $\mathfrak{g}_{\mathbb{C}}$. For a linear functional $\alpha \in \mathfrak{t}_{\mathbb{C}}^{*}$, we define the root space

$$
\mathfrak{g}_{\mathbb{C}}^{\alpha}:=\left\{x \in \mathfrak{g}_{\mathbb{C}}:\left(\forall y \in \mathfrak{t}_{\mathbb{C}}\right)[y, x]=\alpha(y) x\right\}
$$

and write

$$
\Delta:=\Delta\left(\mathfrak{g}_{\mathbb{C}}, \mathfrak{t}_{\mathbb{C}}\right):=\left\{\alpha \in \mathfrak{t}_{\mathbb{C}}^{*} \backslash\{0\}: \mathfrak{g}_{\mathbb{C}}^{\alpha} \neq\{0\}\right\}
$$

for the set of roots of $\mathfrak{g}$.

(b) A root $\alpha \in \Delta$ is called semisimple if $\alpha\left(\left[z, z^{*}\right]\right) \neq 0$ holds for an element $z \in \mathfrak{g}_{\mathbb{C}}^{\alpha}$. In this case, $\left[\mathfrak{g}_{\mathbb{C}}^{\alpha}, \mathfrak{g}_{\mathbb{C}}^{-\alpha}\right]=\mathbb{C}\left[z, z^{*}\right]$ contains a unique element $\alpha^{\vee}$ with $\alpha\left(\alpha^{\vee}\right)=2$ which we call the coroot of $\alpha$. We write $\Delta_{s}$ for the set of semisimple roots and call the roots in $\Delta_{r}:=\Delta \backslash \Delta_{s}$ the solvable roots.

A semisimple root $\alpha$ is called compact if $\alpha^{\vee} \in \mathbb{R}^{+}\left[z, z^{*}\right]$, i.e. if $\alpha\left(\left[z, z^{*}\right]\right)>0$. All other roots are called non-compact. We write $\Delta_{k}$, resp., $\Delta_{p}$ for the set of compact, resp., non-compact roots. We also set $\Delta_{p, s}:=\Delta_{p} \cap \Delta_{s}$.

(c) For each compact root $\alpha \in \Delta_{k}$, the linear mapping $s_{\alpha}: \mathfrak{t} \rightarrow \mathfrak{t}, x \mapsto x-\alpha(x) \alpha^{\vee}$ is a reflection in the hyperplane $\operatorname{ker} \alpha$. We write $\mathcal{W}_{\mathfrak{k}}$ for the group generated by these reflections. It is called the Weyl group of the pair $(\mathfrak{k}, \mathfrak{t})$. According to [12, Prop. VII.2.10], this group is finite. 
Definition 2.9 (a) A subset $\Delta^{+} \subseteq \Delta$ is called a positive system if there exists an element $x_{0} \in i$ with

$$
\Delta^{+}=\left\{\alpha \in \Delta: \alpha\left(x_{0}\right)>0\right\}
$$

and $\alpha\left(x_{0}\right) \neq 0$ holds for all $\alpha \in \Delta$. A positive system $\Delta^{+}$is said to be adapted if for $\alpha \in \Delta_{k}$ and $\beta \in \Delta_{p}^{+}$we have $\beta\left(x_{0}\right)>\alpha\left(x_{0}\right)$ for some $x_{0}$ defining $\Delta^{+}$. In this case, we call $\Delta_{p}^{+}:=\Delta^{+} \cap \Delta_{p}$ an adapted system of positive non-compact roots.

(b) We associate with an adapted system $\Delta_{p}^{+}$of positive non-compact roots the convex cones

$$
C_{\min }:=C_{\min }\left(\Delta_{p}^{+}\right):=\operatorname{cone}\left(\left\{i\left[z_{\alpha}, z_{\alpha}^{*}\right]: z_{\alpha} \in \mathfrak{g}_{\mathbb{C}}^{\alpha}, \alpha \in \Delta_{p}^{+}\right\}\right) \subseteq \mathfrak{t},
$$

and

$$
C_{\max }:=C_{\max }\left(\Delta_{p}^{+}\right):=\left\{x \in \mathfrak{t}:\left(\forall \alpha \in \Delta_{p}^{+}\right) i \alpha(x) \geq 0\right\} .
$$

The structure theoretic concepts introduced above play a crucial role in the analysis of invariant convex subsets. In particular, [12, Thm. VII.3.8] asserts that the existence of a pointed generating $\operatorname{Inn}(\mathfrak{g})$-invariant closed convex cone $W \subseteq \mathfrak{g}$ implies the existence of a compactly embedded Cartan subalgebra (cf. Theorem 2.5), and that, for every compactly embedded Cartan subalgebra $\mathfrak{t}$, there exists an adapted positive system $\Delta_{p}^{+}$ with

$$
C_{\min } \subseteq W \cap \mathfrak{t}=p_{\mathfrak{t}}(W) \subseteq C_{\max }
$$

where $p_{\mathfrak{t}}: \mathfrak{g} \rightarrow \mathfrak{t}$ denotes the projection with kernel $[\mathfrak{t}, \mathfrak{g}]$. Moreover, $W$ is uniquely determined by $W \cap \mathfrak{t}$, the cone $C_{\min }$ is pointed. By [12, Thm. VIII.2.12] (cf. also [12, Thm. VIII.3.7]), for an adapted positive system $\Delta_{p}^{+}$and an admissible Lie algebra, the pointedness of $C_{\min }$ implies that $C_{\min } \subseteq C_{\max }$. Note that

$$
C_{\min }=C_{\min , \mathfrak{z}}+\operatorname{cone}\left(\left\{-i \alpha^{\vee}: \alpha \in \Delta_{p, s}^{+}\right\}\right)
$$

is pointed if and only if $C_{\min , \mathfrak{z}}$ is pointed because $\left\{\alpha^{\vee}: \alpha \in \Delta_{p, s}^{+}\right\}$is a finite subset contained in an open half space. If this condition is satisfied, then [12, Prop. VIII.3.7] shows that

$$
W_{\max }:=\left\{x \in \mathfrak{g}: p_{\mathfrak{t}}\left(\mathcal{O}_{x}\right) \subseteq C_{\max }\right\}
$$

is a generating closed convex invariant cone with $W_{\max } \cap \mathfrak{t}=C_{\max }$, and

$$
W_{\min }:=\left\{x \in \mathfrak{g}: p_{\mathfrak{t}}\left(\mathcal{O}_{x}\right) \subseteq C_{\min }\right\}
$$

is a pointed, closed convex invariant cone with $W_{\min } \cap \mathfrak{t}=C_{\min }$. In general, $W_{\min }$ is not generating. The most extreme situation occurs if $\mathfrak{g}$ is a compact Lie algebra. Then $W_{\min }=\{0\}$ and $W_{\max }=\mathfrak{g}$. 


\section{Elements in Pointed Cones}

In this section, we study elements $x$ in an admissible Lie algebra $\mathfrak{g}=\mathfrak{g}(\mathfrak{l}, V, \mathfrak{z}, \beta)$ for which $\operatorname{co}(x)$ is pointed. Splitting $\mathfrak{l}$ into an ideal $\mathfrak{l}_{0}$ commuting with $V$ and an ideal $\mathfrak{l}_{1}$ acting effectively on $V$, reduces this problem to the two cases, where either $\mathfrak{g}=\mathfrak{l}$ is reductive (Sect. 3.2) or where the reductive subalgebra l acts faithfully on $V$ (Sect. 3.3).

\subsection{General Observations}

Theorem 2.5 provides powerful structural information that is crucial to analyze the subsets $\mathfrak{g}_{\mathrm{c}}$ and $\mathfrak{g}_{\mathrm{co}}$ for non-reductive admissible Lie algebras. Throughout this section we write

$\mathfrak{g}=\mathfrak{u} \rtimes \mathfrak{l}=(\mathfrak{z} \oplus V) \rtimes \mathfrak{l}=\mathfrak{g}(\mathfrak{l}, V, \mathfrak{z}, \beta) \quad$ with $\quad \mathfrak{z}=\mathfrak{z}(\mathfrak{g}) \quad$ and $\quad \beta(v, w)=[v, w]$

The kernel $\mathfrak{l}_{0} \unlhd \mathfrak{l}$ of the representation of $\mathfrak{l}$ on $V$ has a complementary ideal $\mathfrak{l}_{1}$, and $\mathfrak{g}$ is a direct sum

$$
\mathfrak{g}=\mathfrak{g}_{1} \oplus \mathfrak{l}_{0} \quad \text { with } \quad \mathfrak{g}_{1}=\mathfrak{z} \oplus V \oplus \mathfrak{l}_{1}=\mathfrak{g}\left(\mathfrak{l}_{1}, V, \mathfrak{z}, \beta\right)
$$

Any $x \in \mathfrak{g}$ decomposes accordingly as $x=x_{1}+x_{0}$ with $x_{1} \in \mathfrak{g}_{1}$ and $x_{0} \in \mathfrak{l}_{0}$, and

$$
\operatorname{co}(x)=\operatorname{co}\left(x_{1}\right) \times \operatorname{co}\left(x_{0}\right)
$$

implies that

$$
\mathfrak{g}_{\mathrm{co}}=\mathfrak{g}_{1, \mathrm{co}} \times \mathfrak{l}_{0, \mathrm{co}} .
$$

This reduces the description of this set to the two cases, where $\mathfrak{g}$ is reductive or the representation of $\mathfrak{l}$ on $V$ is faithful.

Lemma 3.1 For $x \in \mathfrak{l}$ we put

$$
V_{x, 0}:=\{v \in V:[x, v]=0\} \quad \text { and } \quad V_{x}:=[x, V] \subseteq V
$$

Then the following assertions hold:

(a) If $f \in \mathfrak{z}^{*}$ is such that $\omega:=f \circ \beta$ is a symplectic form, then $V_{x}^{\perp \omega}=V_{x, 0}$ and $V_{x, 0}^{\perp_{\omega}}=V_{x}$.

(b) $V_{x, 0}=\left\{v \in V:\left[v, V_{x}\right]=\{0\}\right\}=V_{x}^{\perp_{\beta}}$ and $V_{x}=\left\{v \in V:\left[v, V_{x, 0}\right]=\{0\}\right\}=$ $V_{x, 0}^{\perp \beta}$

Proof (a) Since $\operatorname{ad}_{V} x:=\left.\operatorname{ad}(x)\right|_{V} \in \mathfrak{s p}(V, \omega)$, we have

$$
V_{x}^{\perp_{\omega}}=\left\{v \in V: \omega\left(v, V_{x}\right)=\{0\}\right\}=\{v \in V: \omega([x, v], V)=\{0\}\}=V_{x, 0} .
$$


Now $V_{x, 0}^{\perp_{\omega}}=V_{x}$ follows from $V_{x} \subseteq V_{x, 0}^{\perp_{\omega}}$ and $\operatorname{dim} V_{x, 0}^{\perp_{\omega}}=\operatorname{dim} V-\operatorname{dim} V_{x, 0}=$ $\operatorname{dim} V_{x}$.

(b) For $v \in V_{x, 0}$, we have $\beta(v,[x, V])=-\beta([x, v], V)=\{0\}$, i.e., $\beta\left(V_{x, 0}, V_{x}\right)=\{0\}$. Moreover, the non-degeneracy of $\beta$ (Corollary 2.6) shows that $\{0\}=\beta(v,[x, V])=\beta([x, v], V)$ implies $[x, v]=0$, i.e., $V_{x}^{\perp_{\beta}}=V_{x, 0}$.

To see that we also have $V_{x, 0}^{\perp_{\beta}} \subseteq V_{x}$, we use (a) and the existence of an $f \in \mathfrak{z}^{*}$ for which $\omega:=f \circ \beta$ is symplectic (Theorem 2.5) to see that $V_{x, 0}^{\perp_{\beta}} \subseteq V_{x, 0}^{\perp_{\omega}}=V_{x}$.

For $y \in V$ and $x=x_{\mathfrak{z}}+x_{V}+x_{\mathfrak{l}} \in \mathfrak{g}$, we have

$$
\begin{aligned}
e^{\operatorname{ad} y} x & =x+[y, x]+\frac{1}{2}[y,[y, x]] \\
& =x+\left[y, x_{V}\right]+\left[y, x_{\mathfrak{l}}\right]+\frac{1}{2}(\operatorname{ad} y)^{2} x_{\mathfrak{l}} \\
& =\underbrace{\left(x_{\mathfrak{z}}+\left[y, x_{V}\right]+\frac{1}{2}\left[y,\left[y, x_{\mathfrak{l}}\right]\right]\right)}_{\in \mathfrak{z}}+\underbrace{\left(x_{V}-\left[x_{\mathfrak{l}}, y\right]\right)}_{\in V}+\underbrace{x_{\mathfrak{l}}}_{\in \mathfrak{l}} .
\end{aligned}
$$

This rather simple formula will be a key tool throughout this paper. We conclude in particular that $e^{\text {ad } y} x \in \mathfrak{z}+\mathfrak{l}$ is equivalent to the vanishing of the $V$-component, i.e., to

$$
x_{V}=\left[x_{\mathfrak{l}}, y\right]
$$

$\operatorname{As} \operatorname{Inn}(\mathfrak{g})=e^{\operatorname{ad} V} \operatorname{Inn}_{\mathfrak{g}}(\mathfrak{l})$,

$$
\mathcal{O}_{x} \cap(\mathfrak{z}+\mathfrak{l}) \neq \varnothing \quad \text { if and only if } \quad x_{V} \in\left[x_{\mathfrak{l}}, V\right]=V_{x} .
$$

If this condition is satisfied, then (3.4), applied with $x_{V}=0$, shows that $e^{\text {ad } V} x \cap(\mathfrak{z}+\mathfrak{l})$ is a single element. For $x \in \mathfrak{z}+\mathfrak{l}$, we thus obtain

$$
\mathcal{O}_{x} \cap(\mathfrak{z}+\mathfrak{l})=\operatorname{Inn}_{\mathfrak{g}}(\mathfrak{l}) x=: \mathcal{O}_{x}^{\mathfrak{l}} .
$$

The following Reduction Theorem can be used to reduce many question concerning the sets $\operatorname{co}(x)$ and $C_{x}$ to elements in reductive Lie algebras.

Theorem 3.2 (Reduction Theorem) Let $\mathfrak{g} \cong \mathfrak{g}(\mathfrak{l}, V, \mathfrak{z}, \beta)$ be an admissible Lie algebra. If $\operatorname{co}(x)$ is pointed, then $\mathcal{O}_{x} \cap(\mathfrak{z}+\mathfrak{l}) \neq \emptyset$.

Proof Write $x=x_{\mathfrak{z}}+x_{V}+x_{\mathfrak{l}}$. From (3.4) we derive in particular that $e^{\text {ad } V_{x_{\mathfrak{l}}, 0}} x=$ $x+\left[V_{x_{l}, 0}, x_{V}\right]$ is an affine subspace. If $\operatorname{co}(x)$ is pointed, this affine subspace is trivial, so that $\left[V_{x_{1}, 0}, x_{V}\right]=\{0\}$. Now $x_{V} \in V_{x_{\mathfrak{l}}}$ follows from Lemma 3.1(b) and the assertion follows from (3.5).

Corollary 3.3 Let $\mathfrak{g} \cong \mathfrak{g}(\mathfrak{l}, V, \mathfrak{z}, \beta)$ be an admissible Lie algebra. Then every adnilpotent element $x \in \mathfrak{g}$ with $\operatorname{co}(x)$ pointed is conjugate under inner automorphisms to an element of $\mathfrak{z}+\mathfrak{s}$ for $\mathfrak{s}=[\mathfrak{l}, \mathfrak{l}]$. Any ad-nilpotent element of $\mathfrak{z}+\mathfrak{l}$ is contained in $\mathfrak{z}+\mathfrak{s}$. 
Proof By Theorem 3.2, we may assume that $x=x_{\mathfrak{z}}+x_{\mathfrak{l}} \in \mathfrak{z}+\mathfrak{l}$, i.e., that $x_{V}=0$. Then ad $x=\operatorname{ad} x_{\mathfrak{l}}$ is nilpotent. Write $x_{\mathfrak{l}}=x_{0}+x_{\mathfrak{s}}$, where $x_{\mathfrak{s}} \in \mathfrak{s}$ is nilpotent and $x_{0} \in \mathfrak{z}(\mathfrak{l})$. Since $\left[x_{0}, x_{\mathfrak{s}}\right]=0, \operatorname{ad}\left(x_{\mathfrak{s}}\right)$ is nilpotent and $\operatorname{ad}\left(x_{0}\right)$ is semisimple because $\mathfrak{z}(\mathfrak{l}) \subseteq \mathfrak{t}_{\mathfrak{l}}$ is compactly embedded, the decomposition $\operatorname{ad}\left(x_{\mathfrak{l}}\right)=\operatorname{ad}\left(x_{0}\right)+\operatorname{ad}\left(x_{\mathfrak{s}}\right)$ is the Jordan decomposition of $\operatorname{ad}\left(x_{\mathfrak{l}}\right)$. Therefore, the nilpotency of this element implies $\operatorname{ad}\left(x_{0}\right)=0$, hence that $x_{0}=0$ because $\mathfrak{z}(\mathfrak{l}) \cap \mathfrak{z}(\mathfrak{g}) \subseteq \mathfrak{l} \cap \mathfrak{z}=\{0\}$. We conclude that $x_{\mathfrak{l}}=x_{\mathfrak{s}} \in \mathfrak{s}$ and thus $x \in \mathfrak{z}+\mathfrak{s}$.

Corollary 3.4 Let $W \subseteq \mathfrak{g}$ be a pointed generating invariant cone. If $x=x_{\mathfrak{z}}+x_{\mathfrak{s}} \in$ $W \cap(\mathfrak{z}(\mathfrak{g})+\mathfrak{s})$ is ad-nilpotent, then $x_{\mathfrak{z}} \in W$ and $x_{\mathfrak{s}} \in W$.

Proof We may assume that the nilpotent element $x_{\mathfrak{s}} \in \mathfrak{s}$ is non-zero, otherwise the assertion is trivial. For any $h \in \mathfrak{s}$ with $\left[h, x_{\mathfrak{s}}\right]=2 x_{\mathfrak{s}}$ (Jacobson-Morozov Theorem, [1, Ch. VIII, §11, Prop. 2]), we have

$x_{\mathfrak{s}}=\lim _{t \rightarrow \infty} e^{-2 t} e^{t \operatorname{ad} h} x \in W \quad$ and $\quad x_{\mathfrak{z}}=\lim _{t \rightarrow \infty} e^{-t \operatorname{ad} h} x \in W$.

Lemma 3.5 If $x$ is a nilpotent element of the pointed generating invariant cone $W \subseteq \mathfrak{g}$ and $x_{\mathfrak{s}} \neq 0$, then there exists a Lie subalgebra $\mathfrak{m} \subseteq \mathfrak{g}$, isomorphic to $\mathfrak{g l}_{2}(\mathbb{R})$, such that $\mathfrak{z}(\mathfrak{m}) \subseteq \mathfrak{z}(\mathfrak{g})$ and $\mathfrak{m} \cap W$ is pointed and generating.

Proof By Corollary 3.3, we may assume that $x=x_{\mathfrak{z}}+x_{\mathfrak{s}} \in \mathfrak{z}(\mathfrak{g})+\mathfrak{s}$ holds for a Levi complement $\mathfrak{s}$. We first choose an $\mathfrak{s l}_{2}(\mathbb{R})$-subalgebra $\mathfrak{s}_{x} \subseteq \mathfrak{s}$ containing $x_{\mathfrak{s}}$ (cf. Proposition B.1). If $x_{\mathfrak{z}} \neq 0$, then

$$
x=x_{\mathfrak{z}}+x_{\mathfrak{s}} \in \mathfrak{m}:=\mathbb{R} x_{\mathfrak{z}}+\mathfrak{s}_{x} \cong \mathfrak{g l}_{2}(\mathbb{R})
$$

Further, Corollary 3.4 implies that $\mathfrak{m} \cap W$ contains $x_{\mathfrak{z}}$ and $x_{\mathfrak{s}}$, hence is generating in $\mathfrak{m}$.

The following observation provides some information on the central part of $\lim (\operatorname{co}(x))$.

Lemma 3.6 For $x=x_{\mathfrak{z}}+x_{V}+x_{\mathfrak{l}} \in \mathfrak{g}=\mathfrak{g}(\mathfrak{l}, V, \mathfrak{z}, \beta)$, we consider the cone

$$
C_{x, \mathfrak{z}}:=C_{x_{\mathfrak{l}, \mathfrak{z}}}:=\operatorname{cone}\left(\left\{\left[y,\left[y, x_{\mathfrak{l}}\right]\right]: y \in V\right\}\right) .
$$

Then

$$
C_{x, \mathfrak{z}} \subseteq \lim (\operatorname{co}(x)) \cap \mathfrak{z} .
$$

Proof For $t \rightarrow \infty$, formula (3.4) leads to

$$
\lim _{t \rightarrow \infty} t^{-2}\left(e^{\operatorname{ad} t y} x\right)=\frac{1}{2}[y,[y, x]]=\frac{1}{2}\left[y,\left[y, x_{\mathfrak{l}}\right]\right] \in \lim (\operatorname{co}(x)) \cap \mathfrak{z} .
$$




\subsection{Reductive Lie Algebras}

If $\mathfrak{g}$ is a simple real Lie algebra and $\mathfrak{k} \subseteq \mathfrak{g}$ a maximal compactly embedded subalgebra, then the existence of a pointed generating invariant cone $W$ implies the existence of a non-zero element $z \in \mathfrak{z}(\mathfrak{k})$, i.e., that $\mathfrak{g}$ is hermitian. If this is the case, then

$$
W_{\min }:=C_{z}
$$

is a minimal invariant cone, the dual cone

$$
W_{\max }:=W_{\min }^{\star}:=\left\{x \in \mathfrak{g}:\left(\forall y \in W_{\min }\right) \kappa(x, y) \geq 0\right\}
$$

with respect to the non-degenerate form $\kappa(x, y)=-\operatorname{tr}(\operatorname{ad} x$ ad $y)$ is a maximal invariant cone containing $W_{\min }$, and any other pointed generating invariant cone $W$ either satisfies

$$
W_{\min } \subseteq W \subseteq W_{\max } \quad \text { or } \quad W_{\min } \subseteq-W \subseteq W_{\max }
$$

depending on whether $z \in W$ or $-z \in W$ (see the Kostant-Vinberg Theorem in [5, Thm. III.4.7], [19]).

Lemma 3.7 [6, Thm. III.9] Let $\mathfrak{g}$ be semisimple and $W \subseteq \mathfrak{g}$ be a pointed generating invariant cone. If $W^{\prime} \supseteq W$ is any pointed invariant cone containing $W$, then every nilpotent element $x \in W^{\prime}$ is contained in $W$.

Proposition 3.8 Let $\mathfrak{g}$ be simple hermitian and $x \in \mathfrak{g}$ with the Jordan decomposition $x=x_{s}+x_{n}$. Then the following are equivalent:

(a) $C_{x}$ is pointed.

(b) $C_{x_{s}}$ and $C_{x_{n}}$ are pointed and, if $x_{s} \neq 0$, then $x_{n} \in C_{x_{s}}$.

(c) $x \in W_{\max } \cup-W_{\max }$, where $W_{\max }$ is a maximal pointed invariant cone.

(d) $\operatorname{co}(x)$ is pointed.

If $x_{s} \neq 0$, then $C_{x}=C_{x_{s}}$.

Proof (a) $\Rightarrow$ (b): If $C_{x}$ is pointed, then $x_{s}, x_{n} \in C_{x}$ follows from Corollary B.2. If $x_{s} \neq 0$, we see that $C_{x_{s}} \subseteq C_{x}$ is a non-trivial invariant cone, and now Lemma 3.7 shows that $x_{n} \in C_{x_{s}}$.

(b) $\Rightarrow$ (a): If $x_{s}=0$ and $C_{x_{n}}$ is pointed, then $C_{x}=C_{x_{n}}$ is pointed. If $x_{s} \neq 0$, we further assume that $x_{n} \in C_{x_{s}}$. Then $x=x_{s}+x_{n} \in C_{x_{s}}$ implies $C_{x} \subseteq C_{x_{s}}$, so that $C_{x}$ is pointed because $C_{x_{s}}$ is assumed to be pointed.

(a) $\Leftrightarrow$ (c) follows from the Kostant-Vinberg Theorem ( [5, Thm. III.4.7], [19]).

That (d) follows from (a) is clear. Suppose that $\operatorname{co}(x)$ is pointed. We may assume that $x \neq 0$, and observe that this implies that $\operatorname{co}(x)$ has interior points. Let $\mathfrak{k} \subseteq \mathfrak{g}$ be maximal compactly embedded. Then $K:=\operatorname{Inn}_{\mathfrak{g}}(\mathfrak{k}) \subseteq \operatorname{Aut}(\mathfrak{g})$ is compact and $\operatorname{co}(x)^{K}=\operatorname{co}(x) \cap \mathfrak{z}(\mathfrak{k})$ contains an interior point $z$. Let $W_{\max }$ be the maximal pointed generating invariant cone containing $z$. 
We claim that $x \in W_{\max }$. The projection $p_{\mathfrak{z}(\mathfrak{k})}: \mathfrak{g} \rightarrow \mathfrak{z}(\mathfrak{k})=\mathfrak{z} \mathfrak{g}(\mathfrak{k})$ is the fixed point projection for the action of the compact group $K$. Therefore it preserves closed convex invariant subsets. This shows that $p_{\mathfrak{z}(\mathfrak{k})}(\operatorname{co}(x)) \subseteq \operatorname{co}(x)$. As $\lim (\operatorname{co}(-z))=-C_{z}$, the subset $p_{\mathfrak{z}(\mathfrak{k})}(\operatorname{co}(x)) \subseteq \mathfrak{z}(\mathfrak{k})=\mathbb{R} z$ must be contained in the half line $[0, \infty) z$. Therefore,

$$
x \in W:=\left\{y \in \mathfrak{g}: p_{\mathfrak{z}(\mathfrak{k})}\left(\mathcal{O}_{y}\right) \subseteq[0, \infty) z\right\}
$$

The right-hand side is a closed convex invariant cone containing $z$. Therefore $x \in$ $W \subseteq W_{\max }$ implies that $C_{x}$ is pointed.

Corollary 3.9 If $\mathfrak{g}$ is simple hermitian, then

$$
\mathfrak{g}_{\mathrm{c}}=\mathfrak{g}_{\mathrm{co}}=W_{\max } \cup-W_{\max }
$$

Proposition 3.10 ( $\mathfrak{g}_{\mathrm{co}}$ for reductive Lie algebras) Let $\mathfrak{g}=\mathfrak{g}_{k}+\mathfrak{g}^{1}+\cdots+\mathfrak{g}^{k}$ be reductive, where $\mathfrak{g}_{k}$ is the maximal compact ideal and the ideals $\mathfrak{g}^{j}$ are simple noncompact. If $\mathfrak{g}^{j}$ is hermitian, we write $W_{\max }^{\mathfrak{g}_{j}}$ for a maximal proper invariant cone in $\mathfrak{g}^{j}$ and otherwise we put $W_{\max }^{\mathfrak{g}_{j}}:=\{0\}$. Then

$$
\mathfrak{g}_{\mathrm{co}}=\mathfrak{g}_{k} \times \prod_{j=1}^{k}\left(W_{\max }^{\mathfrak{g}_{j}} \cup-W_{\max }^{\mathfrak{g}_{j}}\right)
$$

Proof This follows from $\mathfrak{g}_{k, \text { co }}=\mathfrak{g}_{k}$ and Corollary 3.9, which entails $\mathfrak{g}_{\text {co }}^{j}=W_{\max }^{\mathfrak{g}_{j}} \cup$ $-W_{\max }^{\mathfrak{g}_{j}}$ for $j=1, \ldots, k$.

By the preceding proposition, the structure of $\mathfrak{g}_{\mathrm{co}}$ is rather simple and adapted to the decomposition into simple ideals. The subset $\mathfrak{g}_{\mathrm{c}}$ is slightly more complicated. From Corollary B.2, we obtain the following characterization of elements contained in a given invariant cone $W$ in a reductive Lie algebra.

Proposition 3.11 (Reduction to nilpotent and semisimple elements) Let $\mathfrak{g}$ be a reductive Lie algebra and $x \in \mathfrak{g}$ be contained in the invariant cone $W \subseteq \mathfrak{g}$. Write $x=x_{0}+x_{s}+x_{n}$ with $x_{0} \in \mathfrak{z}(\mathfrak{g})$ and the Jordan decomposition $x_{s}+x_{n}$ of the component of $x$ in $[\mathfrak{g}, \mathfrak{g}]$. Then the following are equivalent:

(a) $x \in W$.

(b) $x_{0}+x_{s} \in W$ and $x_{n} \in W$.

Proof For $x \in W$, Corollary B.2, applied to the semisimple Lie algebra [ $\mathfrak{g}, \mathfrak{g}]$, immediately implies (b). Conversely, (b) implies $x=x_{0}+x_{s}+x_{n} \in W+W \subseteq W$.

Corollary 3.12 Let $\mathfrak{g}$ be reductive and $x \in \mathfrak{g}$. We write $x_{n} \in[\mathfrak{g}, \mathfrak{g}]$ for its nilpotent Jordan component and $x_{S}:=x-x_{n}$ for its ad-semisimple Jordan component. Then $C_{x}$ is pointed if and only if $\overline{C_{x_{n}}+C_{x_{s}}}$ is pointed. 
Proof If $C_{x}$ is pointed, then Proposition 3.11 implies that $x_{s}, x_{n} \in C_{x}$, so that $C_{x_{n}}+C_{x_{s}} \subseteq C_{x}$ has pointed closure. The converse follows from $x=x_{n}+x_{s} \in$ $\overline{C_{x_{n}}+C_{x_{s}}}$, which implies $C_{x} \subseteq \overline{C_{x_{n}}+C_{x_{s}}}$.

Lemma 3.13 Suppose that $\mathfrak{g}$ is a reductive Lie algebra and $x \in \mathfrak{g}$ is such that $C_{x}$ is pointed. Then the following are equivalent:

(a) $0 \in \operatorname{co}(x)$.

(b) $x$ is a nilpotent element of $[\mathfrak{g}, \mathfrak{g}]$.

Proof $(\mathrm{a}) \Rightarrow(\mathrm{b})$ : We write $\mathfrak{g}=\mathfrak{z}(\mathfrak{g}) \oplus \mathfrak{g}^{1} \oplus \cdots \mathfrak{g}^{n}$, where the $\mathfrak{g}^{j}$ are simple ideals and accordingly

$$
x=x_{0}+x_{1}+\cdots+x_{n},
$$

so that $\operatorname{co}(x)=\left\{x_{0}\right\} \times \operatorname{co}\left(x_{1}\right) \times \cdots \times \operatorname{co}\left(x_{n}\right)$. Therefore $0 \in \operatorname{co}(x)$ implies that $x_{0}=0$ and that $0 \in \operatorname{co}\left(x_{j}\right)$ for every $j$. It follows that

$$
C_{x}=\sum_{j=1}^{n} C_{x_{j}},
$$

so that all cones $C_{x_{j}} \subseteq \mathfrak{g}^{j}$ are pointed. They are generating if $x_{j} \neq 0$.

It, therefore, suffices to show that, if $\mathfrak{g}$ is simple hermitian and $0 \neq x \in \mathfrak{g}$ is such that $\operatorname{co}(x)$ is pointed with $0 \in \operatorname{co}(x)$, then $x$ is nilpotent. If $x$ is not nilpotent, then $x_{s} \neq 0$. Proposition 3.8(b) shows that $x_{n} \in C_{x_{s}}$, and thus

$$
x=x_{s}+x_{n} \in x_{s}+C_{x_{s}} \subseteq C_{x_{s}}
$$

leads to $\operatorname{co}(x) \subseteq \operatorname{co}\left(x_{s}\right)+C_{x_{s}}$. We claim that $0 \notin \operatorname{co}\left(x_{s}\right)$. The semisimplicity of $x_{s}$ implies that its orbit $\mathcal{O}_{x_{s}}$ is closed (Theorem of Borel-Harish-Chandra, [20, 1.3.5.5]), and since $C_{x_{s}}$ is pointed, the orbit $\mathcal{O}_{x_{s}}$ is admissible in the sense of [12, Def. VII.3.14]. Here we use that $\mathfrak{g} \cong \mathfrak{g}^{*}$ as $\operatorname{Inn}(\mathfrak{g})$-modules, so that adjoint orbits correspond to coadjoint orbits under a linear isomorphism. Next we use [12, Prop. VIII.1.25] to see that, if $p_{\mathfrak{t}}: \mathfrak{g} \rightarrow \mathfrak{t}$ is the projection onto a compactly embedded Cartan subalgebra $\mathfrak{t}$, then

$$
p_{\mathfrak{t}}\left(\operatorname{co}\left(x_{s}\right)\right)=\operatorname{co}\left(x_{s}\right) \cap \mathfrak{t} \subseteq \operatorname{conv}\left(\mathcal{O}_{x_{s}}\right) .
$$

If $\operatorname{co}\left(x_{s}\right)$ contains 0 , it follows that $0 \in \operatorname{conv}\left(\mathcal{O}_{x_{s}}\right)$, but as $\mathcal{O}_{x_{s}}$ is contained in the convex set $C_{x_{s}} \backslash\{0\}$, this is a contradiction.

(b) $\Rightarrow$ (a): If $x$ is nilpotent, then $0 \in \overline{\mathbb{R}_{+} x} \subseteq \overline{\mathcal{O}_{x}}$ (Corollary B.2) implies that $0 \in \operatorname{co}(x)$.

Proposition 3.14 ( $\mathfrak{g}_{\mathrm{c}}$ for reductive admissible Lie algebras) Let $\mathfrak{g}=\mathfrak{g}_{k} \oplus \mathfrak{g}_{p}$ be a reductive Lie algebra, where $\mathfrak{g}_{k}$ is the maximal compact ideal. We write elements $x \in \mathfrak{g}$ accordingly as $x=x_{k}+x_{p}$. Then the following are equivalent: 
(a) $C_{x}$ is pointed.

(b) $C_{x_{p}}$ is pointed and, if $x_{p}$ is nilpotent and $x_{k} \neq 0$, then $x_{k} \notin\left[\mathfrak{g}_{k}, \mathfrak{g}_{k}\right]$.

(c) $\operatorname{co}(x)$ is pointed, and if $0 \in \operatorname{co}(x)$, then $x_{k}=0$ and $x_{p}$ is nilpotent.

Proof We have

$$
\operatorname{co}(x)=\operatorname{co}\left(x_{k}\right)+\operatorname{co}\left(x_{p}\right)
$$

where $\operatorname{co}\left(x_{k}\right)$ is compact. Therefore, $\operatorname{co}(x)$ is pointed if and only if $\operatorname{co}\left(x_{p}\right)$ is pointed, which by Proposition 3.8(d), applied to the simple ideals in $\mathfrak{g}_{p}$, implies that $C_{x_{p}}$ is pointed.

(a) $\Rightarrow$ (b): If $C_{x}$ is pointed, then the argument above shows that $C_{x_{p}}$ is pointed as well. If $x_{p}$ is nilpotent, then $0 \in \operatorname{co}\left(x_{p}\right)$ by Lemma 3.13, so that (3.8) implies that $C_{x_{k}} \subseteq C_{x}$ is also pointed, and this further implies that, if $x_{k} \neq 0$, then $x_{k} \notin\left[\mathfrak{g}_{k}, \mathfrak{g}_{k}\right]$. Here we use that the relative interior of $C_{x_{k}}$ intersects $\mathfrak{z}\left(\mathfrak{g}_{k}\right)$ because the projection $p_{\mathfrak{z}}: \mathfrak{g}_{k} \rightarrow \mathfrak{z}\left(\mathfrak{g}_{k}\right)$ is the fixed point projection for the compact group $\operatorname{Inn}\left(\mathfrak{g}_{k}\right)$.

(b) $\Rightarrow$ (c): Suppose that $C_{x_{p}}$ is pointed. Then

$$
\operatorname{co}(x)=\operatorname{co}\left(x_{k}\right) \times \operatorname{co}\left(x_{p}\right) \subseteq \operatorname{co}\left(x_{k}\right) \times C_{x_{p}},
$$

and since $\operatorname{co}\left(x_{k}\right)$ is compact, $\lim (\operatorname{co}(x)) \subseteq C_{x_{p}}$ is pointed. If $0 \in \operatorname{co}(x)$, then $0 \in$ $\operatorname{co}\left(x_{k}\right)$ and $0 \in \operatorname{co}\left(x_{p}\right)$. As $C_{x_{p}}$ is pointed, Lemma 3.13 implies that $x_{p}$ is nilpotent. If $x_{k} \neq 0$, then $x_{k} \notin\left[\mathfrak{g}_{k}, \mathfrak{g}_{k}\right]$ by (b), contradicting $0 \in \operatorname{co}\left(x_{k}\right) \subseteq x_{k}+\left[\mathfrak{g}_{k}, \mathfrak{g}_{k}\right]$.

(c) $\Rightarrow$ (a): Suppose that $\operatorname{co}(x)$ is pointed and that, if $0 \in \operatorname{co}(x)$, then $x=x_{p}$ is nilpotent. If $0 \notin \operatorname{co}(x)$, then $C_{x}$ is pointed by Lemma A.2. If $0 \in \operatorname{co}(x)$, then $x=x_{p}$ is nilpotent, so that $\operatorname{co}\left(x_{p}\right)=C_{x_{p}}=C_{x}$ is pointed.

Proposition 3.15 (Extension of invariant cones) Suppose that $\mathfrak{g}$ is reductive and quasihermitian, i.e., a direct sum of a compact Lie algebra and hermitian simple ideals. If $C_{x}$ is pointed, then there exists a pointed generating invariant cone $W \subseteq \mathfrak{g}$ containing $x$.

Proof Let $\mathfrak{g}(x)=C_{x}-C_{x} \unlhd \mathfrak{g}$ be the ideal generated by $x$. As $\mathfrak{g}$ is reductive, $\mathfrak{g}=\mathfrak{g}(x) \oplus \mathfrak{g}_{1}$, where $\mathfrak{g}_{1} \unlhd \mathfrak{g}$ is a complementary ideal. If $\mathfrak{g}_{1}$ itself contains a pointed generating invariant cone $W_{1}$, then $C_{x}+W_{1}$ is a pointed generating invariant cone in $\mathfrak{g}$. As $\mathfrak{g}_{1}$ also is quasihermitian, it contains no pointed generating invariant cone if and only if it is compact semisimple (Proposition 3.14). Then we consider a product $B_{x} \times B_{1} \subseteq \mathfrak{g}$, where $B_{x} \subseteq C_{x}$ is a compact base of the pointed cone $C_{x}$, i.e., of the form $f^{-1}(1) \cap C_{x}$ for $f$ in the interior of the dual cone $C_{x}^{\star}$, and $B_{1} \subseteq \mathfrak{g}_{1}$ is a compact invariant 0 -neighborhood. Then $W:=\operatorname{cone}\left(B_{x}+B_{1}\right)$ is a pointed generating invariant cone in $\mathfrak{g}$ containing $C_{x}=\operatorname{cone}\left(B_{x}\right)$.

We shall see below that the preceding proposition does not extend to non-reductive admissible Lie algebras (Example 3.25). 


\subsection{The Characterization Theorem}

We now turn to the non-reductive admissible Lie algebras $\mathfrak{g}=\mathfrak{g}(\mathfrak{l}, V, \mathfrak{z}, \beta)$. With the reduction (3.1) in mind, we may assume that $\mathfrak{l}$ acts faithfully on $V$. We start with a crucial lemma.

For a root $\alpha \in \Delta_{r}$ with root space

$$
\mathfrak{g}_{\mathbb{C}}^{\alpha}=V_{\mathbb{C}}^{\alpha}=\left\{w \in V_{\mathbb{C}}:(\forall x \in \mathfrak{t})[x, w]=\alpha(x) w\right\}
$$

(see [12, Prop. VII.2.5] for $V_{\mathbb{C}}^{\alpha}=\mathfrak{g}_{\mathbb{C}}^{\alpha}$, we consider the closed convex cone

$$
C_{\alpha}:=\operatorname{cone}\left(\left\{i\left[v_{\alpha}, v_{\alpha}^{*}\right]: v_{\alpha} \in V_{\mathbb{C}}^{\alpha}\right\}\right) \subseteq \mathfrak{z},
$$

As $z \mapsto z^{*}$ exchanges $V_{\mathbb{C}}^{\alpha}$ and $V_{\mathbb{C}}^{-\alpha}$, we have

$$
C_{-\alpha}=C_{\alpha}
$$

Lemma 3.16 For any pointed closed convex cone $C_{\mathfrak{z}} \subseteq \mathfrak{z}$,

$$
W_{\mathfrak{l}}:=W_{\mathfrak{l}}\left(C_{\mathfrak{z}}\right):=\left\{x \in \mathfrak{l}:(\forall v \in V)[v,[v, x]] \in C_{\mathfrak{z}}\right\}
$$

is a pointed invariant closed convex cone in $\mathfrak{l}$.

Proof That $W_{\mathfrak{l}}$ is a closed convex invariant cone in $\mathfrak{l}$ follows from the $\mathfrak{l}$-invariance of the bracket $\beta: V \times V \rightarrow \mathfrak{z}$. To see that $W_{\mathfrak{l}}$ is pointed, let $y \in W_{\mathfrak{l}} \cap-W_{\mathfrak{l}}$. Then $[v,[v, y]]=0$ for every $v \in V$. As the map $V \times V \rightarrow \mathfrak{z},(v, w) \mapsto[v,[w, y]]$ is symmetric by

$$
[w,[v, y]]=[[w, v], y]-[[w, y], v]=[v,[w, y]]
$$

polarization shows that $[V,[V, y]]=\{0\}$. Next Corollary 2.6 entails $[V, y]=\{0\}$, so that $y=0$ because the representation of $\mathfrak{l}$ on $V$ is injective. This shows that $W_{\mathfrak{l}}$ is pointed.

Lemma 3.17 For $x \in \mathfrak{t}$, we have $C_{x, \mathfrak{z}}=\overline{\sum_{i \alpha(x)>0} C_{\alpha}}$.

Proof We write $v \in V$ as $v=\sum_{\alpha \in \Delta_{r}^{+}} v_{\alpha}-v_{\alpha}^{*}$ with $v_{\alpha} \in V_{\mathbb{C}}^{\alpha}$, where $\Delta_{r}^{+} \subseteq \Delta_{r}$ is any positive system. We then find for $x \in \mathfrak{t}$ :

$$
[v, x]=\sum_{\alpha \in \Delta_{r}^{+}}-\alpha(x)\left(v_{\alpha}+v_{\alpha}^{*}\right)
$$


and thus

$$
\begin{aligned}
{[v,[v, x]] } & =\sum_{\beta, \alpha \in \Delta_{r}^{+}}-\alpha(x)\left[v_{\beta}-v_{\beta}^{*}, v_{\alpha}+v_{\alpha}^{*}\right]=\sum_{\alpha \in \Delta_{r}^{+}}-\alpha(x)\left(\left[v_{\alpha}, v_{\alpha}^{*}\right]-\left[v_{\alpha}^{*}, v_{\alpha}\right]\right) \\
& =\sum_{\alpha \in \Delta_{r}^{+}}-2 \alpha(x)\left[v_{\alpha}, v_{\alpha}^{*}\right]=\sum_{\alpha \in \Delta_{r}^{+}} 2 i \alpha(x) i\left[v_{\alpha}, v_{\alpha}^{*}\right] .
\end{aligned}
$$

This shows that $i \alpha(x)>0$ implies that $C_{\alpha} \subseteq C_{x, \mathfrak{z}}$. As $-i \alpha(x) C_{-\alpha}=i \alpha(x) C_{\alpha}$ by (3.9), the cone $C_{x, \mathfrak{z}}$ is generated by the cones $C_{\alpha}$ for $i \alpha(x)>0, \alpha \in \Delta_{r}$.

Proposition 3.18 Let $\Delta_{r}^{+} \subseteq \Delta_{r}$ be a positive system and

$$
C_{\mathfrak{z}} \supseteq C_{\min , \mathfrak{z}}:=\overline{\sum_{\alpha \in \Delta_{r}^{+}} C_{\alpha}}=\operatorname{cone}\left(\left\{i\left[v_{\alpha}, v_{\alpha}^{*}\right]: \alpha \in \Delta_{r}^{+}, v_{\alpha} \in V_{\mathbb{C}}^{\alpha}\right\}\right)
$$

be a pointed closed convex cone in $\mathfrak{z}$. Then

$$
W_{\mathfrak{l}}:=W_{\mathfrak{l}}\left(C_{\mathfrak{z}}\right):=\left\{x \in \mathfrak{l}:(\forall v \in V)[v,[v, x]] \in C_{\mathfrak{z}}\right\}
$$

is a pointed generating closed convex invariant cone in $\mathfrak{l}$ with

$$
W_{\mathfrak{l}} \cap \mathfrak{t}_{\mathfrak{l}}=\left(i \Delta_{r}^{+}\right)^{\star}
$$

By [12, Thm. VII.2.7], any $x \in \mathfrak{t}_{\mathfrak{l}}$ for which some Hamiltonian function $H_{x}^{f}, f \in C_{\mathfrak{z}}^{\star}$, is positive definite is contained in the interior of $W_{\mathfrak{l}}$.

Proof In view of Lemma 3.16, it remains to show that $W_{\mathfrak{l}}$ is generating. First we observe that

$$
W_{\mathfrak{l}}=\left\{x \in \mathfrak{l}: C_{x, \mathfrak{z}} \subseteq C_{\mathfrak{z}}\right\}
$$

If $x \in \mathfrak{t}_{\mathfrak{l}}$ is such that $i \alpha(x)>0$ for all $\alpha \in \Delta_{r}^{+}$, then $C_{x, \mathfrak{z}}=C_{\min , \mathfrak{z}}$ by Lemma 3.17. Therefore,

$$
W_{\mathfrak{l}} \cap \mathfrak{t}_{\mathfrak{l}} \supseteq\left(i \Delta_{r}^{+}\right)^{\star}
$$

and this cone has inner points. This implies that $W_{\mathfrak{l}}$ is generating because $\operatorname{Inn}(\mathfrak{l})\left(W_{\mathfrak{l}} \cap \mathfrak{t}_{\mathfrak{l}}\right)$ contains inner points. This in turn follows from the fact that the differential of the map

$$
\Phi: \mathfrak{l} \times \mathfrak{t}_{\mathfrak{l}} \rightarrow \mathfrak{l}, \quad \Phi(x)(y):=e^{\operatorname{ad} x} y
$$

is surjective if $y \in \mathfrak{t}_{\mathfrak{l}}$ is regular.

If $x \in \mathfrak{t}_{\mathfrak{l}}$ is such that $i \alpha(x)<0$ for some $\alpha \in \Delta_{r}^{+}$, then $C_{\alpha} \subseteq-C_{\min , \mathfrak{z}} \subseteq-C_{\mathfrak{z}}$ and the pointedness of $C_{\mathfrak{z}}$ thus shows that $C_{x, \mathfrak{z}} \nsubseteq C_{\mathfrak{z}}$. We conclude in particular that $x \notin W_{\mathfrak{l}}$, so that $W_{\mathfrak{l}} \cap \mathfrak{t} \subseteq\left(i \Delta_{r}^{+}\right)^{\star}$, and thus (3.10) follows. 
Remark 3.19 In the context of the preceding proposition, we assume, in addition, that $\Delta_{p}^{+}$is adapted and consider the closed convex cone

$$
W:=\left\{x \in \mathfrak{g}: \mathcal{O}_{x} \subseteq C_{\mathfrak{z}}+V+W_{\mathfrak{l}}\right\}
$$

Then the pointedness of $C_{\min , \mathfrak{z}}$ implies the pointedness of $C_{\min }$ and hence that $C_{\min } \subseteq$ $C_{\max }$ ([12, Thm. VIII.2.12]). As

$$
\operatorname{Inn}(\mathfrak{g}) W_{\mathfrak{l}}=e^{\operatorname{ad} V} W_{\mathfrak{l}} \subseteq C_{\mathfrak{z}}+V+W_{\mathfrak{l}}
$$

follows from (3.4), we have $C_{\mathfrak{z}}+W_{\mathfrak{l}} \subseteq W$. For

$$
\mathfrak{z} W:=C_{\mathfrak{z}}-C_{\mathfrak{z}} \supseteq C_{\min , \mathfrak{z}}-C_{\min , \mathfrak{z}}=[V, V],
$$

the cone $W$ is clearly contained in the ideal $\mathfrak{g}_{W}:=\mathfrak{g}\left(\mathfrak{l}, V, \mathfrak{z}_{W}, \beta\right)$. Further,

$$
C_{\mathfrak{z}}+\left(W_{\mathfrak{l}} \cap \mathfrak{t}_{\mathfrak{l}}\right) \subseteq W \cap \mathfrak{t} \subseteq C_{\mathfrak{z}}+\left(W_{\mathfrak{l}} \cap \mathfrak{t}_{\mathfrak{l}}\right)
$$

implies that

$$
W \cap \mathfrak{t}=C_{\mathfrak{z}}+\left(W_{\mathfrak{l}} \cap \mathfrak{t}_{\mathfrak{l}}\right),
$$

and this cone is generating in $\mathfrak{t}_{W}:=\mathfrak{t} \cap \mathfrak{g}_{W}$, which in turn implies that $W$ is generating in $\mathfrak{g}_{W}$. By construction, $H(W) \subseteq V$ is trivial by Lemma 2.7(b), so that $W$ is pointed.

Theorem 3.20 (Characterization Theorem) Let $\mathfrak{g}=\mathfrak{g}(\mathfrak{l}, V, \mathfrak{z}, \beta)$ be admissible such that the representation of $\mathfrak{l}$ on $V$ is faithful. For $x=x_{\mathfrak{z}}+x_{V}+x_{\mathfrak{l}} \in \mathfrak{g}$ we consider the $\mathfrak{z}$-valued Hamiltonian function

$$
H_{x}^{\mathfrak{z}}: V \rightarrow \mathfrak{z}, \quad H_{x}^{\mathfrak{z}}(v):=p_{\mathfrak{z}}\left(e^{\operatorname{ad} v} x\right)=x_{\mathfrak{z}}+\left[v, x_{V}\right]+\frac{1}{2}\left[v,\left[v, x_{\mathfrak{l}}\right]\right]
$$

and put

$$
\operatorname{co}_{\mathfrak{z}}(x):=\overline{\operatorname{conv}}\left(H_{x}^{\mathfrak{z}}(V)\right) .
$$

Then

(a) $\operatorname{co}(x)$ is pointed if and only if $\operatorname{co}_{\mathfrak{z}}(x)$ is pointed, i.e., $\mathfrak{g}_{\mathrm{co}}=\left\{x \in \mathfrak{g}: \operatorname{co}_{\mathfrak{z}}(x)\right.$ pointed $\}$.

(b) $C_{x}$ is pointed if and only if $\operatorname{co}_{\mathfrak{z}}(x)$ is pointed and, if $x_{\mathfrak{l}}$ is nilpotent, then $\operatorname{co}_{\mathfrak{z}}(x)$ is contained in a pointed cone.

We shall see in Example 3.25 below that the pointedness of $C_{x}$ does in general not imply the existence of a pointed generating invariant cone $W$ containing $x$.

Proof (a) If $\operatorname{co}(x)$ is pointed, then we may assume that $x=x_{\mathfrak{z}}+x_{\mathfrak{l}}$ by Theorem 3.2. Note that $H_{x}^{\mathfrak{z}}$ only depends on the orbit $e^{\text {ad } V} x$. Now $\operatorname{co}\left(x_{\mathfrak{l}}\right)=\operatorname{co}(x)-x_{\mathfrak{z}}$ is also pointed. 
That the cone $C_{x, \mathfrak{z}}=C_{x_{\mathfrak{l}} \mathfrak{z}}=\operatorname{co}_{\mathfrak{z}}\left(x_{\mathfrak{l}}\right)$ is pointed follows from $C_{x, \mathfrak{z}} \subseteq \mathfrak{z} \cap \lim (\operatorname{co}(x))$ (Lemma 3.6).

Suppose, conversely, that $\operatorname{co}_{\mathfrak{z}}(x)$ is pointed. As

$$
C_{x, \mathfrak{z}} \subseteq \lim \left(\operatorname{co}_{\mathfrak{z}}(x)\right),
$$

by Lemma 3.6, the cone $C_{x, \mathfrak{z}}$ is pointed. For $V_{x_{\mathfrak{l}}, 0}:=\left\{v \in V:\left[x_{\mathfrak{l}}, v\right]=0\right\}$, the set $H_{x}^{\mathfrak{z}}\left(V_{x_{\mathfrak{l}}, 0}\right)=x_{\mathfrak{z}}+\left[V_{x_{\mathfrak{l}}, 0}, x_{V}\right]$ is an affine space. Our assumption implies that it is trivial, so that $\left[x_{V}, V_{x_{1}, 0}\right]=\{0\}$ and thus $x_{V} \in\left[x_{\mathfrak{l}}, V\right]$ by Lemma 3.1(b). Hence, $\mathcal{O}_{x} \cap(\mathfrak{z} \oplus \mathfrak{s}) \neq \varnothing$ follows from (3.5). We may, therefore, assume that $x_{V}=0$. Then $x=x_{\mathfrak{z}}+x_{\mathfrak{l}}$ and $\operatorname{co}(x)=x_{\mathfrak{z}}+\operatorname{co}\left(x_{\mathfrak{l}}\right)$. It therefore suffices to show that $\operatorname{co}\left(x_{\mathfrak{l}}\right)$ is pointed if the cone $C_{x_{\mathfrak{l}}, \mathfrak{z}}$ is pointed.

By Lemma 3.16,

$$
W_{\mathfrak{l}}:=\left\{y \in \mathfrak{l}:(\forall v \in V)[v,[v, y]] \in C_{x_{\mathfrak{l}, \mathfrak{z}}}\right\}
$$

is a pointed invariant cone in $\mathfrak{l}$. As $x_{\mathfrak{l}} \in W_{\mathfrak{l}}$, we obtain

$$
\operatorname{co}\left(x_{\mathfrak{l}}\right) \subseteq C_{x_{\mathfrak{l}, \mathfrak{z}}}+V+W_{\mathfrak{l}}
$$

Therefore the linear subspace $H\left(\operatorname{co}\left(x_{\mathfrak{l}}\right)\right)$ is an ideal of $\mathfrak{g}$ contained in $V$, hence trivial (Lemma 2.7(b)). This shows that $\operatorname{co}\left(x_{\mathfrak{l}}\right)$ is pointed.

(b) Suppose that $C_{x}$ is pointed. Then $\operatorname{co}(x)$ is pointed and thus $\operatorname{co}_{\mathfrak{z}}(x)$ is pointed by (a). Suppose that $x_{\mathfrak{l}}$ is nilpotent. We have to show that $\operatorname{co}_{\mathfrak{z}}(x)$ is contained in a pointed cone. From

$$
C_{x, \mathfrak{z}} \subseteq \lim (\operatorname{co}(x)) \subseteq C_{x}
$$

(Lemma 3.6) it follows that $C_{x, \mathfrak{z}}$ is pointed. As we have seen in (a), we may assume that $x_{V}=0$, so that $x=x_{\mathfrak{z}}+x_{\mathfrak{l}}$. Further, the nilpotency of $x_{\mathfrak{l}}$ implies that it is contained in $\mathfrak{s}$ (Corollary 3.3). Moreover, $0 \in \operatorname{co}\left(x_{\mathfrak{l}}\right)$ by Lemma 3.13, so that $C_{x} \supseteq$ $\operatorname{co}(x)=x_{\mathfrak{z}}+\operatorname{co}\left(x_{\mathfrak{l}}\right) \ni x_{\mathfrak{z}}$. We conclude that

$$
\operatorname{co}_{\mathfrak{z}}(x)=x_{\mathfrak{z}}+C_{x_{\mathfrak{l}, \mathfrak{z}}}=x_{\mathfrak{z}}+C_{x, \mathfrak{z}} \subseteq C_{x}
$$

is contained in the pointed cone $C_{x} \cap \mathfrak{z}$.

Conversely, suppose that $\operatorname{co}_{\mathfrak{z}}(x)$ is pointed and further that $x_{V}=0$ by (a) and Theorem 3.2.

- If $\operatorname{co}_{\mathfrak{z}}(x)$ is contained in a pointed cone $D_{x} \subseteq \mathfrak{z}$, then

$$
\operatorname{co}(x) \subseteq D_{x}+V+\operatorname{col}_{\mathfrak{l}}\left(x_{\mathfrak{l}}\right)
$$

In the proof of (a) we have seen that $C_{x_{\mathfrak{l}}, \mathfrak{z}} \subseteq \lim \left(\operatorname{co}_{\mathfrak{z}}(x)\right)$ is pointed, and that $x_{\mathfrak{l}}$ is contained in the pointed invariant cone $W_{\mathfrak{l}} \subseteq \mathfrak{l}$ from (3.12). Therefore $C_{x} \subseteq$ $D_{x}+V+W_{\mathfrak{l}}$ shows that $H\left(C_{x}\right) \unlhd \mathfrak{g}$ is an ideal contained in $V$, hence trivial (Lemma 2.7(b)). 
- If $\operatorname{co}_{\mathfrak{z}}(x)$ is not contained in a pointed cone, then we assume that $x_{\mathfrak{l}}$ is not nilpotent. We show that $C_{x}$ is pointed by verifying that $0 \notin \operatorname{co}(x)$ and applying Lemma A.2. As $\operatorname{co}(x)=x_{\mathfrak{z}}+\operatorname{co}\left(x_{\mathfrak{l}}\right)$, we have to show that $-x_{\mathfrak{z}} \notin \operatorname{co}\left(x_{\mathfrak{l}}\right)$. We claim that $0 \notin \operatorname{col}_{\mathfrak{l}}\left(x_{\mathfrak{l}}\right)$. In fact, if $x_{\mathfrak{l}}=y_{s}+y_{n}$ is the Jordan decomposition, where $y_{n} \in[\mathfrak{l}, \mathfrak{l}]$ is nilpotent and $y_{s}=x_{\mathfrak{l}}-y_{n}$, then $y_{s}, y_{n} \in W_{\mathfrak{l}}$ (Proposition 3.11). This shows that

$$
\mathcal{O}_{x_{\mathfrak{l}}}^{L} \subseteq \mathcal{O}_{y_{s}}^{L}+W_{\mathfrak{l}} \quad \text { implies } \quad \operatorname{col}\left(x_{\mathfrak{l}}\right) \subseteq \operatorname{col}\left(y_{s}\right)+W_{\mathfrak{l}} \subseteq W_{\mathfrak{l}}
$$

As $y_{s} \neq 0$ by assumption and $W_{\mathfrak{l}}$ is pointed by (a), $0 \notin \operatorname{cor}_{\mathfrak{l}}\left(y_{s}\right)$ by Lemma 3.13, and this implies that $0 \notin \operatorname{cor}_{\mathfrak{l}}\left(x_{\mathfrak{l}}\right)$. Finally we observe that

$$
\operatorname{co}\left(x_{\mathfrak{l}}\right) \subseteq \mathfrak{z}+V+\operatorname{col}\left(x_{\mathfrak{l}}\right)
$$

and by the preceding argument, this convex set intersects $\mathfrak{z}$ trivially. Therefore, $-x_{\mathfrak{z}} \notin \operatorname{co}\left(x_{\mathfrak{l}}\right)$.

Remark 3.21 (a) From (3.13) we derive in particular that the pointedness of $\operatorname{co}(x)$ implies the pointedness of $\operatorname{co}_{\mathfrak{l}}\left(x_{\mathfrak{l}}\right) \subseteq \mathfrak{l}$, i.e., $p_{\mathfrak{l}}\left(\mathfrak{g}_{\mathrm{co}}\right) \subseteq \mathfrak{l}_{\mathrm{co}}$.

(b) For $x=x_{\mathfrak{z}}+x_{\mathfrak{l}} \in \mathfrak{z}+\mathfrak{l}$, we have

$$
\operatorname{co}_{\mathfrak{z}}(x)=x_{\mathfrak{z}}+C_{x, \mathfrak{z}},
$$

which is pointed if and only if $C_{x, \mathfrak{z}}$ is pointed. If this is the case, then $\operatorname{co}_{\mathfrak{z}}(x)$ is contained in a pointed cone if and only if

$$
\left(x_{\mathfrak{z}}+C_{x, \mathfrak{z}}\right) \cap-C_{x, \mathfrak{z}} \subseteq\{0\},
$$

which means that the intersection either is empty or $x_{\mathfrak{z}}=0$ (cf. Lemma A.3).

Remark 3.22 Let $f \in \mathfrak{z}^{*}$, considered as a linear functional on $\mathfrak{g}=\mathfrak{g}(\mathfrak{l}, V, \mathfrak{z}, \beta)$ via $f(z, v, x):=f(z)$. Then $f$ is fixed under the coadjoint action of $\operatorname{Inn}_{\mathfrak{g}}(\mathfrak{l})$, so that its coadjoint orbit is

$$
\mathcal{O}_{f}=f \circ \operatorname{Inn}(\mathfrak{g})=f \circ e^{\mathrm{ad} V}
$$

For $x=x_{\mathfrak{z}}+x_{V}+x_{\mathfrak{l}} \in \mathfrak{g}$ we therefore have $\mathcal{O}_{f}(x)=f\left(e^{\operatorname{ad} V} x\right)=f \circ H_{x}^{\mathfrak{z}}(V)$. This implies that

$$
B\left(\mathcal{O}_{f}\right):=\left\{x \in \mathfrak{g}: \inf \mathcal{O}_{f}(x)>-\infty\right\}=\left\{x \in \mathfrak{g}: \inf f\left(\operatorname{co}_{\mathfrak{z}}(x)\right)>-\infty\right\} .
$$

For elements $x=x_{\mathfrak{z}}+x_{\mathfrak{l}} \in \mathfrak{z}+\mathfrak{l}$, it follows that

$$
x \in B\left(\mathcal{O}_{f}\right) \text { if and only if } f \in C_{x, \mathfrak{z}}^{\star} .
$$


Corollary 3.23 Let $\mathfrak{g}:=\mathfrak{h s p}_{2 n}(\mathbb{R})$ be the Jacobi-Lie algebra of inhomogeneous polynomials of degree $\leq 2$ on the symplectic vector space $\left(\mathbb{R}^{2 n}, \omega\right)$, endowed with the Poisson bracket. Then the following assertions hold:

(a) $\operatorname{co}(x)$ is pointed if and only if the corresponding function $H_{x}$ on $\mathbb{R}^{2 n}$ is semibounded, i.e., bounded from below or above.

(b) $C_{x}$ is pointed if and only if $x$ or $-x$ satisfies

(i) $H_{x}$ is bounded from below, and

(ii) if $x_{\mathfrak{l}} \neq 0$ is nilpotent, then $H_{x} \geq 0$.

Proof We shall obtain this is a special case of Theorem 3.20. The Jacobi algebra is admissible of the form $\mathfrak{g}(\mathfrak{l}, V, \mathfrak{z}, \beta)$ with

$$
\begin{aligned}
& \mathfrak{l}=\mathfrak{s p}_{2 n}(\mathbb{R}), \quad V=\mathbb{R}^{2 n}, \quad \mathfrak{z}=\mathbb{R} \text { and } \\
& \quad \beta=\omega, \quad \omega\left(\left(\mathbf{p}_{1}, \mathbf{q}_{1}\right),\left(\mathbf{p}_{2}, \mathbf{q}_{2}\right)\right)=\mathbf{p}_{1} \mathbf{q}_{2}-\mathbf{p}_{2} \mathbf{q}_{1} .
\end{aligned}
$$

Further,

$$
H_{x}^{\mathfrak{z}}(v)=x_{\mathfrak{z}}+\omega\left(v, x_{V}\right)+\frac{1}{2} \omega\left(x_{\mathfrak{l}} \cdot v, v\right)=H_{x}(v)
$$

is the Hamiltonian function on $(V, \omega)$, corresponding to $x \in \mathfrak{g}$.

(a) We conclude that $\operatorname{co}_{\mathfrak{z}}(x) \subseteq \mathfrak{z}=\mathbb{R}$ is the closed convex hull of the range of $H_{x}^{\mathfrak{z}}$. This is a pointed convex set if and only if $H_{x}$ is semibounded.

(b) means that $C_{x}$ is pointed if and only if $H_{x}$ is semibounded and, if $x_{\mathfrak{l}}$ is nilpotent, then $H_{x} \geq 0$ (if $H_{x}$ is bounded from below) or $H_{x} \leq 0$ (if $H_{x}$ is bounded from above). So (b) follows from Theorem 3.20(b).

Example 3.24 For $V=\mathbb{R}^{2}$ and $\mathfrak{s}=\mathfrak{s p}_{2}(\mathbb{R})=\mathfrak{s l}_{2}(\mathbb{R})$, the Hamiltonian function associated with

$$
x=\left(\begin{array}{cc}
a & b \\
c & -a
\end{array}\right) \quad \text { is given by } \quad H_{x}(q, p)=b p^{2}-c q^{2}+2 a p q .
$$

We have $H_{x} \geq 0$ if and only if $b,-c \geq 0$ and $a^{2} \leq-b c$. Then $x$ is either elliptic or nilpotent, where the latter is equivalent to $a^{2}=-b c$.

Accordingly, an element $x=x_{\mathfrak{z}}+x_{V}+x_{\mathfrak{s}} \in \mathfrak{h s p}_{2}(\mathbb{R})$ with $H_{x_{\mathfrak{s}}} \geq 0$ generates a pointed cone if either $x_{\mathfrak{s}}$ is elliptic, i.e., positive definite, or if $x_{\mathfrak{s}}$ is nilpotent and $H_{x} \geq 0$.

Example 3.25 (Elements in $\mathfrak{g}_{c}$, not contained in a pointed generating cone) We consider the Lie algebra $\mathfrak{g}=\mathfrak{g}(\mathfrak{l}, V, \mathfrak{z}, \beta)$, where

$$
\mathfrak{l}=\mathbb{R}^{2}, \quad V=V_{1} \oplus V_{2} \oplus V_{1,2} \quad \text { with } \quad V_{1}=V_{2}=\mathbb{C}, V_{1,2}=\mathbb{C}^{2}, \quad \mathfrak{z}=\mathbb{R}^{2},
$$

and the action of $\mathfrak{l}$ on $V$ is given by

$$
\left(x_{1}, x_{2}\right) \cdot\left(z_{1}, z_{2}, z_{3}, z_{4}\right)=\left(i x_{1} z_{1}, i x_{2} z_{2}, i\left(x_{1}+x_{2}\right) z_{3}, i\left(x_{1}+x_{2}\right) z_{4}\right) .
$$


With $\varepsilon_{j}\left(x_{1}, x_{2}\right)=i x_{j}$, this means that

$$
\Delta=\Delta_{r}=\left\{ \pm \varepsilon_{1}, \pm \varepsilon_{2}, \pm\left(\varepsilon_{1}+\varepsilon_{2}\right)\right\}
$$

We define $\beta: V \times V \rightarrow \mathfrak{z}$ by

$$
\beta(\mathbf{z}, \mathbf{w}):=\left(\operatorname{Im}\left(\overline{z_{1}} w_{1}\right)+\operatorname{Im}\left(\overline{z_{3}} w_{3}\right), \operatorname{Im}\left(\overline{z_{2}} w_{2}\right)+\operatorname{Im}\left(\overline{z_{4}} w_{4}\right)\right) \in \mathbb{R}^{2}=\mathfrak{z} .
$$

With $V^{[\alpha]}:=V \cap\left(V_{\mathbb{C}}^{\alpha}+V_{\mathbb{C}}^{-\alpha}\right)$, we then have

$$
V^{\left[\varepsilon_{1}\right]}=V_{1}, \quad V^{\left[\varepsilon_{2}\right]}=V_{2} \quad \text { and } \quad V^{\left[\varepsilon_{1}+\varepsilon_{2}\right]}=V_{1,2}
$$

For $y:=(-1,0) \in \mathfrak{l}$, we obtain with $i \varepsilon_{1}(y)=1$ :

$$
C_{\varepsilon_{1}}=\operatorname{cone}\left\{[v,[v, y]]: v \in V_{1}\right\}=\operatorname{cone}\left\{\beta(-i v, v): v \in V_{1}\right\}=[0, \infty) \mathbf{e}_{1} .
$$

We likewise obtain $C_{\varepsilon_{2}}=[0, \infty) \mathbf{e}_{2}$, and

$$
C_{\varepsilon_{1}+\varepsilon_{2}}=\operatorname{cone}\left\{\beta(-i v, v): v \in V_{1,2}\right\}=[0, \infty) \mathbf{e}_{1}+[0, \infty) \mathbf{e}_{2}
$$

Therefore $\Delta_{r}^{+}:=\left\{\varepsilon_{1}, \varepsilon_{2}, \varepsilon_{1}+\varepsilon_{2}\right\}$ is an adapted positive system for which

$$
C_{\min }=[0, \infty) \mathbf{e}_{1}+[0, \infty) \mathbf{e}_{2} \subseteq \mathfrak{z} \subseteq C_{\max }
$$

is pointed. The invariant cone

$$
W_{\min }:=\left\{x \in \mathfrak{g}: p_{\mathfrak{t}}\left(\mathcal{O}_{x}\right) \subseteq C_{\min }\right\}
$$

satisfies $W_{\min } \cap \mathfrak{t} \subseteq C_{\min }$, so that $H\left(W_{\text {min }}\right) \unlhd \mathfrak{g}$ is an ideal contained in $V$. As $\beta: V \times V \rightarrow \mathfrak{z}$ is non-degenerate, this ideal is trivial and therefore $W_{\min }$ is pointed. Now [12, Lemma VIII.3.22] implies that $\mathfrak{g}$ is admissible.

Consider the element $x:=(1,-1) \in \mathfrak{l}$. As $\left(\varepsilon_{1}+\varepsilon_{2}\right)(x)=0, i \varepsilon_{1}(x)=-1$ and $i \varepsilon_{2}(x)=1$, the cone

$$
C_{x, \mathfrak{z}}=C_{\varepsilon_{2}}-C_{\varepsilon_{1}}=\operatorname{cone}\left(\mathbf{e}_{2},-\mathbf{e}_{1}\right)
$$

is pointed, so that $C_{x}$ is pointed by the Characterization Theorem 3.20.

We claim that there exists no pointed generating invariant cone $W \subseteq \mathfrak{g}$ containing $x$. Suppose that $W$ is such a cone. Then there exists an adapted positive system $\Delta_{r}^{+}$ with $C_{\min , \mathfrak{z}} \subseteq W$ [12, Thm. VII.3.8]. As $C_{x, \mathfrak{z}} \subseteq C_{x} \subseteq W$ (Lemma 3.6), we must have $\varepsilon_{2},-\varepsilon_{1} \in \Delta_{r}^{+}$. If $\varepsilon_{1}+\varepsilon_{2}$ is positive, then

$$
C_{\varepsilon_{1}} \subseteq C_{\varepsilon_{1}+\varepsilon_{2}} \subseteq C_{\min , \mathfrak{z}} \quad \text { and } \quad-C_{\varepsilon_{1}}=C_{-\varepsilon_{1}} \subseteq C_{\min , \mathfrak{z}}
$$


contradict the pointedness of $C_{\min , \mathfrak{z}}$. If $\varepsilon_{1}+\varepsilon_{2}$ is negative, then

$$
-C_{\varepsilon_{2}} \subseteq C_{-\varepsilon_{1}-\varepsilon_{2}} \subseteq C_{\min , \mathfrak{z}} \quad \text { and } \quad C_{\varepsilon_{2}} \subseteq C_{\min , \mathfrak{z}}
$$

contradict the pointedness of $C_{\min , \mathfrak{z}}$. Hence there exists no pointed generating invariant cone $W$ containing $x$.

In the preceding example it was important that $\operatorname{dim} \mathfrak{z}>1$. We have the following positive result for the Jacobi-Lie algebra, where $\mathfrak{z}=\mathbb{R}$.

Proposition 3.26 If $\mathfrak{g}=\mathfrak{h s p}_{2 n}(\mathbb{R})=\mathfrak{g}\left(\mathfrak{s p}_{2 n}(\mathbb{R}), \mathbb{R}^{2 n}, \mathbb{R}, \omega\right)$ and $x \in \mathfrak{g}$ is such that $C_{x}$ is pointed, then $x$ is contained in a pointed generating invariant cone $W \subseteq \mathfrak{g}$.

Proof In view of Corollary 3.23, we may assume that the Hamiltonian function $H_{x}$ is bounded from below. We write $x=x_{\mathfrak{z}}+x_{V}+x_{\mathfrak{l}}$ with $x_{\mathfrak{z}} \in \mathfrak{z}=\mathbb{R}, x_{V} \in V=\mathbb{R}^{2 n}$ and $x_{\mathfrak{l}} \in \mathfrak{s p}_{2 n}(\mathbb{R})$. If $x_{\mathfrak{l}}$ is nilpotent, then even $H_{x} \geq 0$, so that

$$
x \in W:=\left\{y \in \mathfrak{g}: H_{y} \geq 0\right\}
$$

and $W$ is a pointed generating invariant cone in $\mathfrak{g}$.

We may therefore assume that $x_{\mathfrak{l}}$ is not nilpotent. By the Reduction Theorem 3.2, we may further assume that $x_{V}=0$. If $H_{x} \geq 0$, then $x \in W$; so we assume that $x_{\mathfrak{z}}=\min H_{x}(V)=H_{x}(0)<0$. We now show that

$$
W \cap-C_{x}=\{0\}
$$

As $x \in \mathfrak{z}+V+W_{\mathfrak{l}}$ for $W_{\mathfrak{l}}:=W \cap \mathfrak{l}$ (the cone of non-negative quadratic forms), the invariance of the set on the right implies $C_{x} \subseteq \mathfrak{z}+V+W_{\mathfrak{l}}$. We conclude that $W \cap-C_{x} \subseteq \mathfrak{z}+V$ is a pointed invariant cone. As $e^{\text {ad } V} x=x+[V, x]$ for $x \in \mathfrak{z}+V$, it follows that $W \cap-C_{x} \subseteq \mathfrak{z}$. We thus obtain

$$
W \cap-C_{x}=(W \cap \mathfrak{z}) \cap\left(-C_{x} \cap \mathfrak{z}\right) .
$$

If $C_{x, \mathfrak{z}}=\{0\}$, then $x_{\mathfrak{l}}=0$ and $x=x_{\mathfrak{z}} \in-W$. So we may also assume that $C_{x, \mathfrak{z}} \neq\{0\}$. As $H_{x}$ is bounded from below, $C_{x} \supseteq C_{x, \mathfrak{z}}=[0, \infty)$, so that we must have $C_{x} \cap \mathfrak{z} \subseteq[0, \infty)$, whence

$$
W \cap-C_{x} \subseteq[0, \infty) \cap(-\infty, 0]=\{0\}
$$

Now [12, Prop. V.1.7] implies that the invariant cone $W+C_{x} \subseteq \mathfrak{g}$ is closed and pointed. It is generating because $W$ is generating.

\section{Affine Pairs}

In this section, we turn to affine pairs related to invariant cones. We refer to the introduction for the motivation to study such pairs. 
Definition 4.1 (Affine pair) Let $\mathfrak{g}$ be a finite dimensional real Lie algebra and $W \subseteq \mathfrak{g}$ a pointed invariant cone. We call $(x, h) \in \mathfrak{g} \times \mathfrak{g}$ an affine pair for the cone $W$ if

$$
x \in W \quad \text { and } \quad[h, x]=x .
$$

For an affine pair, the subalgebra $\mathbb{R} h+\mathbb{R} x$ is isomorphic to the non-abelian 2dimensional Lie algebra $\mathfrak{a f f}(\mathbb{R})$; hence the name. As this Lie algebra is solvable, ad $x$ is nilpotent [4, Prop. 5.4.14].

\subsection{Invariance of W Under One-Parameter Groups of Outer Automorphisms}

On $\mathfrak{g}=\mathfrak{g}(\mathfrak{l}, V, \mathfrak{z}, \beta)$ we consider the canonical derivation $D_{\text {can }}$, defined by

$$
D_{\text {can }}(z, v, x):=\left(z, \frac{1}{2} v, 0\right)
$$

The derivation $2 D_{\text {can }}$ corresponds to the $\mathbb{Z}$-grading of $\mathfrak{g}$, defined by

$$
\mathfrak{g}_{0}=\mathfrak{l}, \quad \mathfrak{g}_{1}=V \quad \text { and } \quad \mathfrak{g}_{2}=\mathfrak{z} .
$$

In the Existence Theorem 4.7 below, the one-parameter group $e^{\mathbb{R} D}$ with $D \in D_{\text {can }}+$ ad $\mathfrak{g}$ leaves an invariant cone $W$ in $\mathfrak{g}$ invariant if and only if $e^{\mathbb{R} D_{\text {can }}}$ does. The following proposition characterizes the cones $W$ for which this is the case.

Proposition 4.2 For a pointed generating invariant cone $W \subseteq \mathfrak{g}=\mathfrak{g}(\mathfrak{l}, V, \mathfrak{z}, \beta)$ and a compactly embedded Cartan subalgebra $\mathfrak{t} \subseteq \mathfrak{g}$, the following are equivalent:

(a) $e^{\mathbb{R} D_{\text {can }} W}=W$.

(b) $p_{\mathfrak{z}}(W) \subseteq W$ and $p_{\mathfrak{l}}(W) \subseteq W$.

(c) $e^{\mathbb{R} D_{\text {can }}}(W \cap \mathfrak{t})=W \cap \mathfrak{t}$.

If $W$ satisfies these conditions, then

$$
W \cap(\mathfrak{z}+\mathfrak{l})=W_{\mathfrak{z}}+W_{\mathfrak{l}} \quad \text { for } \quad W_{\mathfrak{z}}:=W \cap \mathfrak{z} \quad \text { and } \quad W_{\mathfrak{l}}:=W \cap \mathfrak{l} \text {, }
$$

and the Reduction Theorem 3.2 implies that

$$
W=e^{\operatorname{ad} V} \cdot\left(W_{\mathfrak{z}}+W_{\mathfrak{l}}\right)=\overline{\operatorname{Inn}(\mathfrak{g}) \cdot\left(W_{\mathfrak{z}}+W_{\mathfrak{l}}\right)}
$$

(cf. [12, Thm. VII.3.29]), showing that $W$ is uniquely determined by the two cones $W_{\mathfrak{z}}$ and $W_{\mathfrak{l}}$.

Proof $(\mathrm{a}) \Rightarrow(\mathrm{b})$ : For $x=x_{\mathfrak{z}}+x_{V}+x_{\mathfrak{l}} \in W$ we have for $t \rightarrow \infty$

$$
\begin{aligned}
& e^{-t}\left(e^{t D_{\text {can }}} x\right)=x_{\mathfrak{z}}+e^{-t / 2} x_{V}+e^{-t} x_{\mathfrak{l}} \rightarrow x_{\mathfrak{z}} \quad \text { and } \\
& e^{-t D_{\text {can }}} x=e^{-t} x_{\mathfrak{z}}+e^{-t / 2} x_{V}+x_{\mathfrak{l}} \rightarrow x_{\mathfrak{l}} .
\end{aligned}
$$


(b) $\Rightarrow$ (a): Let $x=x_{\mathfrak{z}}+x_{V}+x_{\mathfrak{l}} \in W$. Then Theorem 3.2 implies the existence of $\varphi \in \operatorname{Inn}(\mathfrak{g})$ with $y:=\varphi(x) \in \mathfrak{z}+\mathfrak{l}$. Then $y_{\mathfrak{z}} \in W$ and $y_{\mathfrak{l}} \in W$ by (a). Therefore, $e^{t D_{\text {can }}} y=e^{t} y_{\mathfrak{z}}+y_{\mathfrak{l}} \in W$ for $t \in \mathbb{R}$. Now

$$
e^{t D_{\mathrm{can}}} x=e^{t D_{\mathrm{can}}} \varphi^{-1}(y)=e^{t D_{\mathrm{can}}} \varphi^{-1} e^{-t D_{\mathrm{can}}}\left(e^{t D_{\mathrm{can}}} y\right) \in \operatorname{Inn}(\mathfrak{g}) W=W .
$$

This shows that $e^{t D_{\text {can }}} W \subseteq W$ for every $t \in \mathbb{R}$. As $e^{\mathbb{R} D_{\text {can }}}$ is a group, this implies (a).

(a) $\Rightarrow$ (c) follows from $D_{\text {can }}(\mathfrak{t}) \subseteq \mathfrak{t}$.

(c) $\Rightarrow$ (a): As $W$ is pointed and generating, [12, Thm. VII.3.29] yields $W=$ $\overline{\operatorname{Inn}(\mathfrak{g})(W \cap \mathfrak{t})}$, and the invariance of $W$ under $e^{\mathbb{R} D_{\text {can }}}$ follows from (c).

Example 4.3 Not every pointed generating invariant cone satisfies $p_{\mathfrak{z}}(W) \nsubseteq W_{\mathfrak{z}}$ : For the Jacobi algebra

$$
\mathfrak{g}=\mathfrak{h} \mathfrak{s p}_{2}(\mathbb{R})=\mathfrak{g}\left(\mathfrak{s p}_{2}(\mathbb{R}), \mathbb{R}^{2}, \mathbb{R}, \omega\right) \quad \text { and } \quad \omega(\mathbf{x}, \mathbf{y})=x_{1} y_{2}-x_{2} y_{1},
$$

we have a 2-dimensional compactly embedded Cartan subalgebra

$$
\mathfrak{t}=\mathfrak{z} \oplus \mathfrak{t}_{\mathfrak{s}} \cong \mathbb{R}^{2} \quad \text { and } \quad \mathcal{W}_{\mathfrak{k}}=\left\{\mathrm{id}_{\mathfrak{t}}\right\}
$$

Up so sign, there is a unique positive system $\Delta^{+}$(which is adapted). Then

$$
C_{\min }=C_{\min , \mathfrak{z}} \oplus C_{\min , \mathfrak{s}}
$$

is a quarter plane and

$$
C_{\max }=\mathbb{R} \oplus C_{\max , \mathfrak{s}}=\mathbb{R} \oplus C_{\min , \mathfrak{s}}
$$

is a half plane. Any pointed generating closed convex cone $W_{\mathfrak{t}} \subseteq \mathfrak{t}$ with

$$
C_{\min } \subseteq W_{\mathfrak{t}} \subseteq C_{\max }
$$

is of the form $W_{\mathfrak{t}}=W \cap \mathfrak{t}$ for a pointed generating invariant cone $W \subseteq \mathfrak{g}$ because the Weyl group $\mathcal{W}_{\mathfrak{k}}$ is trivial [12, Thm. VIII.3.21]. In particular, we may have $p_{\mathfrak{z}}\left(W_{\mathfrak{t}}\right)=$ $\mathfrak{z}(\mathfrak{g}) \nsubseteq W_{\mathfrak{t}}$. Therefore, we do not always have $p_{\mathfrak{z}}(W) \subseteq W$.

Remark 4.4 Although the conditions in Proposition 4.2 are not always satisfied, this is the case for many naturally constructed cones.

Let $\mathfrak{g}=\mathfrak{g}(\mathfrak{l}, V, \mathfrak{z}, \beta)$ be an admissible Lie algebra and $D \in \operatorname{der}(\mathfrak{g})$. We assume that the representation of $\mathfrak{l}$ on $V$ is faithful. The cone $W$, constructed from a pointed cone $C_{\mathfrak{z}} \subseteq \mathfrak{z}$ in Remark 3.19 is generated by $C_{\mathfrak{z}}+W_{\mathfrak{l}}$ and satisfies $p_{\mathfrak{z}}(W) \subseteq C_{\mathfrak{z}} \subseteq W$ and $p_{\mathfrak{l}}(W) \subseteq W_{\mathfrak{l}} \subseteq W$. Therefore $W$ is invariant under $e^{\mathbb{R} D_{\text {can }}}$.

More generally, any derivation $D \in \operatorname{der}(\mathfrak{g})$ with $\mathfrak{l} \subseteq \operatorname{ker} D$ and $e^{\mathbb{R} D} C_{\mathfrak{z}}=C_{\mathfrak{z}}$ satisfies $e^{\mathbb{R} D} W=W$ because

$$
W=e^{\operatorname{ad} V}(W \cap(\mathfrak{z}+\mathfrak{l}))=\operatorname{Inn}(\mathfrak{g})(W \cap(\mathfrak{z}+\mathfrak{l}))
$$

follows from the Reduction Theorem 3.2. 


\subsection{Extending Nilpotent Elements to Affine Pairs}

Let $W \subseteq \mathfrak{g}$ be a pointed generating invariant cone. In this section, we consider a nilpotent element $x \in W$ and ask for the existence of a derivation $D \in \operatorname{der}(\mathfrak{g})$ with

$$
D x=x \quad \text { and } \quad e^{\mathbb{R} D} W=W .
$$

Note that the latter condition implies that $W$ is an invariant cone in the extended Lie algebra $\mathfrak{g}_{D}:=\mathfrak{g} \rtimes \mathbb{R} D$ and $(x, D)$ is an affine pair for $W$.

This problem is trivial for semisimple Lie algebras:

Remark 4.5 (a) If $\mathfrak{g}$ is semisimple, $W \subseteq \mathfrak{g}$ is an invariant cone, and $x \in \mathfrak{g}$ is nilpotent, then the Jacobson-Morozov Theorem [1, Ch. VIII, §11, Prop. 2] implies the existence of elements $h, y \in \mathfrak{g}$ with

$$
[h, x]=x, \quad[h, y]=-y \quad \text { and } \quad[x, y]=h .
$$

Then $D:=\operatorname{ad} h$ is a derivation with $D x=x$ and $e^{\mathbb{R} D} W=W$.

(b) If $\mathfrak{g}=\mathfrak{z}(\mathfrak{g}) \oplus[\mathfrak{g}, \mathfrak{g}]$ is reductive and $x=x_{\mathfrak{z}}+x_{\mathfrak{s}}$ with $0 \neq x_{\mathfrak{z}} \in \mathfrak{z}(\mathfrak{g})$ and $x_{\mathfrak{s}} \in[\mathfrak{g}, \mathfrak{g}]$, then it cannot be reproduced with inner derivations. For any derivation $D$ on $\mathfrak{g}$ there exists an endomorphism $D_{\mathfrak{z}}$ of $\mathfrak{z}(\mathfrak{g})$ and an element $h \in[\mathfrak{g}, \mathfrak{g}]$ with

$$
D(z+x)=D_{\mathfrak{z}}(z)+[h, x] \quad \text { for } \quad z \in \mathfrak{z}(\mathfrak{g}), x \in \mathfrak{g},
$$

so that $D x=x$ is equivalent to $D_{\mathfrak{z}} x_{\mathfrak{z}}=x_{\mathfrak{z}}$ and $\left[h, x_{\mathfrak{s}}\right]=x_{\mathfrak{s}}$. If $W \ni x$ is a pointed generating invariant cone, then the nilpotency of $x_{\mathfrak{s}}$ implies that $\mathbb{R}_{+} x_{\mathfrak{s}} \subseteq \mathcal{O}_{x_{\mathfrak{s}}}$ (Corollary B.2), so that $x_{\mathfrak{z}}, x_{\mathfrak{s}} \in W$. Putting $D_{\mathfrak{z}}:=\mathrm{id}_{\mathfrak{z}(\mathfrak{g})}$, we then have $D x=x$ and at least $e^{\mathbb{R} D}\left(W_{\mathfrak{z}}+W_{\mathfrak{s}}\right) \subseteq W$ for $W_{\mathfrak{z}}:=W \cap \mathfrak{z}(\mathfrak{g})$ and $W_{\mathfrak{s}}:=W \cap[\mathfrak{g}, \mathfrak{g}]$.

As the maximal cone $W_{\max } \subseteq \mathfrak{g}$ contains $\mathfrak{z}(\mathfrak{g})$, it is invariant under $e^{\mathbb{R} D}$ for any $D \in \operatorname{der}(\mathfrak{g})$.

The following lemma provides crucial information that we shall need below to explore the existence of Euler derivations on $\mathfrak{g}(\mathfrak{l}, V, \mathfrak{z}, \beta)$, i.e., a diagonalizable derivation with $\operatorname{Spec}(D) \subseteq\{0, \pm 1\}$.

Lemma 4.6 Let $\mathfrak{l}$ be a reductive quasihermitian Lie algebra and $\mathfrak{s} \subset \mathfrak{l}$ be a semisimple subalgebra invariant under a compactly embedded Cartan subalgebra $\mathfrak{t}_{\mathfrak{l}}$ such that every simple ideal in $\mathfrak{s}$ is hermitian of tube type. Let $(V, \omega)$ be a symplectic l-module of convex type and consider the subspaces

$$
V_{\mathrm{eff}, \mathfrak{s}}:=\operatorname{span}(\mathfrak{s} . V) \text { and } V_{\mathrm{fix}, \mathfrak{s}}:=\{v \in V: \mathfrak{s} \cdot v=\{0\}\}
$$

Then the following assertions hold:

(a) $V=V_{\mathrm{eff}, \mathfrak{s}} \oplus V_{\mathrm{fix}, \mathfrak{s}}$ is an $\omega$-orthogonal direct sum of $\mathfrak{s}$-submodules and the submodule $V_{\mathrm{eff}, \mathfrak{s}}$ is a symplectic module of convex type for $\mathfrak{s}$.

(b) For every Euler element $h \in \mathfrak{s}$ for which $\mathfrak{s}_{ \pm 1}(h)$ generate $\mathfrak{s}$, the operator 2 ad $h$ defines an antisymplectic involution on $V_{\mathrm{eff}, \mathfrak{s}}$. 
Proof (a) Since $(V, \omega)$ is a symplectic l-module of convex type with respect to the action of $\mathfrak{l}$, the invariant cone

$$
W_{V, \mathfrak{l}}=\{x \in \mathfrak{l}:(\forall v \in V) \omega(x . v, v) \geq 0\}
$$

is generating, hence intersects the Cartan subalgebra $\mathfrak{t}_{\mathfrak{l}}$. Therefore $(V, \omega)$ also is a symplectic module of convex type for the reductive subalgebra $\mathfrak{l}^{\prime}:=\mathfrak{t}_{\mathfrak{l}}+\mathfrak{s}$. The edge $\mathfrak{l}_{1}$ of the generating invariant cone

$$
W_{V, \mathfrak{l}^{\prime}}=\left\{x \in \mathfrak{l}^{\prime}:(\forall v \in V) \omega(x . v, v) \geq 0\right\}
$$

is the kernel of the representation of $\mathfrak{l}^{\prime}$ on $V$, hence an ideal. We write

$$
\mathfrak{l}^{\prime}=\mathfrak{l}_{1} \oplus \mathfrak{l}_{2}
$$

with a complementary ideal $\mathfrak{l}_{2}$. Then

$$
W_{V, \mathfrak{l}^{\prime}}=\mathfrak{l}_{1} \oplus W_{V, \mathfrak{l}_{2}} \text { with } W_{V, \mathfrak{l}_{2}} \text { pointed and generating. }
$$

As $\mathfrak{s}=\left[\mathfrak{l}^{\prime}, \mathfrak{l}^{\prime}\right]$ adapts to the decomposition (4.3),

$$
\mathfrak{s}=\mathfrak{s}_{1} \oplus \mathfrak{s}_{2} \quad \text { with } \quad \mathfrak{s}_{j}:=\mathfrak{s} \cap \mathfrak{l}_{j}
$$

Now

$$
W_{V, \mathfrak{s}}=\{x \in \mathfrak{s}:(\forall v \in V) \omega(x . v, v) \geq 0\}=\mathfrak{s}_{1} \oplus W_{V, \mathfrak{s}_{2}},
$$

where the pointed cone $W_{V, \mathfrak{s}_{2}}=W_{V, \mathfrak{l}_{2}} \cap \mathfrak{s}_{2}$ is also generating because it contains $W_{\min , \mathfrak{s}_{2}}$. Therefore, $(V, \omega)$ is a symplectic $\mathfrak{s}_{2}$-module of convex type. As $V$ is a semisimple $\mathfrak{s}$-module,

$$
V=V_{\mathrm{eff}, \mathfrak{s}} \oplus V_{\mathrm{fix}, \mathfrak{s}}=V_{\mathrm{eff}, \mathfrak{s}_{2}} \oplus V_{\mathrm{fix}, \mathfrak{s}_{2}}
$$

where $V_{\text {eff, } \mathfrak{s}}$ is a symplectic $\mathfrak{s}_{2}$-module of convex type because $W_{V, \mathfrak{s}_{2}}=W_{V_{\mathrm{eff}}, \mathfrak{s}}$ is pointed and generating [13, Prop. II.5].

(b) Let $h \in \mathfrak{s}$ be an Euler element for which $\mathfrak{s}_{ \pm 1}(h)$ generate $\mathfrak{s}$. We decompose $\mathfrak{s}$ into a direct sum $\bigoplus_{j=1}^{n} \mathfrak{s}_{j}$ of simple ideals, which are hermitian of tube type because they possess Euler elements [9, Prop. 3.11(b)]. By [17, Thm. 2.14], we can decompose $V_{\text {eff, } s}$ into a direct sum $\bigoplus_{j=1}^{n} V_{j}$ of $\mathfrak{s}$-submodules such that $\mathfrak{s}_{k}$ acts trivially on $V_{j}$ for $k \neq j$. In particular, each $\left(V_{j}, \omega\right)$ is a symplectic $\mathfrak{s}_{j}$-module of convex type. We decompose $h$ as $h=\sum_{j=1}^{n} h_{j}$, with $h_{j} \in \mathfrak{s}_{j}$. Then each $h_{j}$ is an Euler element in $\mathfrak{s}_{j}$. Hence [17, Lem. 3.4] implies that, for each $j$, the operator $2 \mathrm{ad} h_{j}$ defines an antisymplectic involution on $V_{j}$, and thus $2 \mathrm{ad} h$ defines an antisymplectic involution on $V_{\mathrm{eff}, \mathfrak{s}}$. 
Theorem 4.7 (Existence Theorem) Let $\mathfrak{g}=\mathfrak{g}(\mathfrak{l}, V, \mathfrak{z}, \beta)$ be an admissible Lie algebra and $x=x_{\mathfrak{z}}+x_{\mathfrak{l}} \in \mathfrak{z}+\mathfrak{l}$ be an ad-nilpotent element for which $\operatorname{co}(x)$ is pointed. Then there exists a derivation $D \in D_{\text {can }}+$ ad $\mathfrak{g}$ with $D x=x$ and

$$
\operatorname{Spec}(D) \subseteq\left\{0, \pm \frac{1}{2}, \pm 1\right\}
$$

Any invariant cone $W$ generated by $W_{\mathfrak{l}}:=W \cap \mathfrak{l}$ and a central cone $W_{\mathfrak{z}} \subseteq \mathfrak{z}$ satisfies $e^{\mathbb{R} D} W=W$.

Recall that, by Corollary 3.3, any ad-nilpotent element $x$ with $C_{x}$ pointed is conjugate to an element of $\mathfrak{z}+\mathfrak{s}$.

Proof By Corollary 3.3, $x_{\mathfrak{s}}:=x_{\mathfrak{l}} \in \mathfrak{s}=[\mathfrak{l}, \mathfrak{l}]$. The nilpotent elements $x_{\mathfrak{s}} \in \mathfrak{s}$ is contained in the sum of all non-compact (hence hermitian) simple ideals and $C_{x_{\mathfrak{s}}}$ is pointed because the pointedness of

$$
\operatorname{co}_{\mathfrak{s}}\left(x_{\mathfrak{s}}\right) \subseteq \operatorname{co}\left(x_{\mathfrak{s}}\right)=\operatorname{co}(x)-x_{\mathfrak{z}}
$$

implies that $C_{x_{\mathfrak{s}}} \subseteq \mathfrak{s}$ is pointed (Proposition 3.14). Now the classification of nilpotent elements in invariant cones implies the existence of an ad-diagonalizable element $h_{\mathfrak{s}} \in \mathfrak{s}$ with

$$
\left[h_{\mathfrak{s}}, x_{\mathfrak{s}}\right]=x_{\mathfrak{s}} \quad \text { and } \quad \operatorname{Spec}\left(\operatorname{ad} h_{\mathfrak{s}}\right)=\left\{0, \pm \frac{1}{2}, \pm 1\right\}
$$

[6, Lemma IV.7]. The element $h_{\mathfrak{s}}$ is obtained using a system of strongly orthogonal restricted roots to show that $x_{\mathfrak{s}}$ is contained in a subalgebra $\mathfrak{b} \cong \mathfrak{s l}_{2}(\mathbb{R})^{r}$ in which $h_{\mathfrak{s}}$ is an Euler element (but in general not in $\mathfrak{s}$ or $\mathfrak{g}$ ). For any element $h_{\mathfrak{s}} \in \mathfrak{s}$ with $\left[h_{\mathfrak{s}}, x_{\mathfrak{s}}\right]=x_{\mathfrak{s}}(\mathrm{cf}$. Remark 4.5), the derivation

$$
D:=D_{\text {can }}+\operatorname{ad} h_{\mathfrak{s}}
$$

(see (4.2)) then satisfies

$$
D x=D\left(x_{\mathfrak{z}}+x_{\mathfrak{s}}\right)=x_{\mathfrak{z}}+\left[h_{\mathfrak{s}}, x_{\mathfrak{s}}\right]=x_{\mathfrak{z}}+x_{\mathfrak{s}}=x .
$$

As $D_{\text {can }} h_{\mathfrak{s}}=0$, the derivation $D$ is diagonalizable because both summands commute and are diagonalizable. The eigenvalues on $\mathfrak{s}$ are contained in $\left\{0, \pm \frac{1}{2}, \pm 1\right\}$ by (4.4) and $\mathfrak{z} \subseteq \operatorname{ker}(D-\mathbf{1})$.

Let

$$
V=V_{0}\left(h_{\mathfrak{s}}\right)+V_{1 / 2}\left(h_{\mathfrak{s}}\right)+V_{-1 / 2}\left(h_{\mathfrak{s}}\right)
$$

denote the $h_{\mathfrak{s}}$-eigenspaces in $V$ (Lemma 4.6). Then the corresponding eigenvalues of $D$ on $V$ are $\frac{1}{2}, 1$ and 0 . This completes the proof of the first assertion.

If the invariant cone $W \subseteq \mathfrak{g}$ is generated by $W_{\mathfrak{l}}$ and a central cone $W_{\mathfrak{z}}$, then the invariance of both cones under $e^{\mathbb{R} D_{\text {can }}}$ implies that $e^{\mathbb{R} D_{\text {can }}} W=W$, hence that $e^{\mathbb{R} D} W=W$ follows from $D \in D_{\text {can }}+$ ad $\mathfrak{g}$. 
Remark 4.8 If the ideal $\mathfrak{s}^{\prime}$ of $\mathfrak{s}$ generated by a nilpotent element $x_{\mathfrak{s}} \in \mathfrak{s}$ contains only simple summands of tube type, then their restricted root systems are of type $\left(C_{r}\right)$ and never of type $\left(B C_{r}\right)$ [3, pp. 587-588]. Therefore, [6, Lemma IV.7] actually provides an Euler element $h_{\mathfrak{s}}$ of $\mathfrak{s}^{\prime}$, and hence also of $\mathfrak{s}$.

\subsection{Euler Derivations}

Definition 4.9 We call $D \in \operatorname{der}(\mathfrak{g})$ an Euler derivation if $D$ is diagonalizable with

$$
\operatorname{Spec}(D) \subseteq\{-1,0,1\}
$$

In this section we ask, for a nilpotent element $x$ for which $C_{x}$ is pointed for an Euler derivation $D$ satisfying $D x=x$. Recall that the latter relation implies that $x$ is nilpotent (cf. Definition 4.1).

Remark 4.10 If $\mathfrak{g}$ is simple hermitian, then every derivation of $\mathfrak{g}$ is inner and an Euler derivation exists if and only if $\mathfrak{g}$ is of tube type [9, Prop. 3.11(b)]. Therefore, Euler derivations $D$ with $D x=x$ need not exist. Concrete examples of hermitian Lie algebras without Euler derivations are $\mathfrak{s u}_{p, q}(\mathbb{C})$ for $p \neq q$. If, however, $\mathfrak{g}$ is hermitian of tube type, then there exists an Euler element $h$ with $[h, x]=x$ (Remark 4.8).

Remark 4.11 Let $\mathfrak{g}=\mathfrak{g}(\mathfrak{l}, V, \mathfrak{z}, \beta)$ be an admissible Lie algebra and $D^{\prime} \in \operatorname{der}(\mathfrak{g})$ an Euler derivation. Then $D^{\prime}$ is conjugate under $e^{\text {ad } \mathfrak{u}}=e^{\text {ad } V}$ to an Euler derivation $D$ such that

$$
D(\mathfrak{l}) \subseteq \mathfrak{l}, \quad D(\mathfrak{z}(\mathfrak{l}))=\{0\}
$$

[16, Thm. 8.1.5]. Then $D(\mathfrak{z}) \subseteq \mathfrak{z}$ and $D(\mathfrak{u}) \subseteq \mathfrak{u}$ imply that

$$
D(V)=D([\mathfrak{l}, \mathfrak{u}]) \subseteq[D \mathfrak{l}, \mathfrak{u}]+[\mathfrak{l}, D \mathfrak{u}] \subseteq[\mathfrak{l}, \mathfrak{u}]=V
$$

Hence there exists an element $h \in \mathfrak{s}:=[\mathfrak{l}, \mathfrak{l}]$ and endomorphisms $D_{\mathfrak{z}} \in \operatorname{End}(\mathfrak{z})$ and $D_{V} \in \operatorname{End}(V)$ with

$$
D(z, v, x)=\left(D_{\mathfrak{z}} z, D_{V} v+[h, v],[h, x]\right) \quad \text { for } \quad(z, v, x) \in \mathfrak{g} .
$$

It follows in particular that $D$ preserves all ideals of $\mathfrak{l}$.

Proposition 4.12 Let $\mathfrak{g}$ be an admissible non-reductive Lie algebra with $\mathfrak{z}(\mathfrak{g}) \subseteq[\mathfrak{g}, \mathfrak{g}]$. If $h$ is an Euler element of $\mathfrak{g}$, then $[h, \mathfrak{r}]=\{0\}$.

Proof By [16, Thm. 8.1.5], we may assume that $\mathfrak{g}=\mathfrak{g}(\mathfrak{l}, V, \mathfrak{z}, \beta)$ with $[h, \mathfrak{l}] \subseteq \mathfrak{l}$. We show that this implies that $h \in \mathfrak{z}+\mathfrak{s}$ for $\mathfrak{s}=[\mathfrak{l}, \mathfrak{l}]$. Write $h=h_{\mathfrak{z}}+h_{V}+h_{\mathfrak{l}}$. Then $[h, \mathfrak{l}] \subseteq \mathfrak{l}$ implies that

$$
\left[h_{V}, \mathfrak{l}\right] \subseteq \mathfrak{l} \cap[V, \mathfrak{l}] \subseteq \mathfrak{l} \cap V=\{0\}
$$


As $\mathfrak{z} V(\mathfrak{l}) \subseteq \mathfrak{z}_{V}\left(\mathfrak{t}_{\mathfrak{l}}\right)=\{0\}$ (Theorem 2.5(d)), this implies $h_{V}=0$, i.e., $h \in \mathfrak{z}+\mathfrak{l}$ and, therefore, ad $h \in \operatorname{ad}(\mathfrak{l})$. We may therefore assume that $h \in \mathfrak{l}$.

Write $h=h_{0}+h_{1}$ with $h_{0} \in \mathfrak{z}(\mathfrak{l})$ and $h_{1} \in \mathfrak{s}$. These two summands commute and $h_{1}$ is an Euler element in $\mathfrak{s}$. As elements of $\mathfrak{z}(\mathfrak{l})$ acts with purely imaginary spectrum on $V$, we further obtain $h_{0}=0$, so that $h \in \mathfrak{s}$.

As the radical $\mathfrak{r}$ of $\mathfrak{g}$ is

$$
\mathfrak{r}=\mathfrak{z}+V+\mathfrak{z}(\mathfrak{l})=[V, V]+V+\mathfrak{z}(\mathfrak{l})
$$

and $[h, \mathfrak{z}(\mathfrak{l})]=\{0\}$, it remains to show that $[h, V]=\{0\}$. Let $f \in \mathfrak{z}^{*}$ be such that $(V, f \circ \beta)$ is a symplectic l-module of convex type. By applying Lemma 4.6 to the ideal $\mathfrak{s}^{\prime} \subset \mathfrak{l}$ generated by $\mathfrak{s}_{ \pm 1}(h)$ and the $\mathfrak{l}$-module $\left(V_{\mathfrak{s}^{\prime}}, f \circ \beta\right)$, we see that $2 \operatorname{ad}(h)$ acts by an involution on $V_{\mathfrak{s}^{\prime}}$. As $h$ is an Euler element of $\mathfrak{g}$, it follows that $V_{\mathfrak{s}^{\prime}}=\{0\}$, hence that $\left[\mathfrak{s}^{\prime}, V\right]=\{0\}$, and thus in particular $[h, V]=\{0\}$.

Example 4.13 Let $(V, \omega)$ be a finite dimensional symplectic vector space. We consider $\mathfrak{g}=\mathfrak{g}(\mathfrak{s p}(V, \omega), V, \mathbb{R}, \omega)$ Then the Levi complement is $\mathfrak{s p}(V, \omega)$, and all Euler elements $h$ in $\mathfrak{s p}(V, \omega)$ are conjugate to $\frac{1}{2} \tau$, where $\tau$ is an antisymplectic involution on $V$ [9, Prop. 3.11(b)]. Therefore the eigenvalues of $h$ on $\mathfrak{g}$ contain $\pm \frac{1}{2}$ and both Lie algebras contain no Euler element.

The following result shows in particular that solvable admissible Lie algebras do not even possess Euler derivations.

Proposition 4.14 Let $\mathfrak{g}$ be a solvable admissible Lie algebra with $\mathfrak{z}(\mathfrak{g}) \subset[\mathfrak{g}, \mathfrak{g}]$. Then there exists no non-zero Euler derivation on $\mathfrak{g}$.

Proof Let $D \in \operatorname{der}(\mathfrak{g})$ be an Euler derivation. By [16, Thm. 8.1.5], we may assume that $\mathfrak{g}=\mathfrak{g}(\mathfrak{l}, V, \mathfrak{z}, \beta)$ as in Theorem 2.5 with $D(\mathfrak{l}) \subseteq \mathfrak{l}$. As $\mathfrak{g}$ is solvable, the Lie algebra $\mathfrak{l}$ is abelian, so that $[17$, Thm. 2.27] shows that $D(\mathfrak{l})=\{0\}$. In particular, $V=[\mathfrak{l}, \mathfrak{u}]$ is $D$-invariant, so that

$$
D(z, v, x)=\left(D_{\mathfrak{z}} z, D_{V} v, 0\right) \text { for }(z, v, x) \in \mathfrak{z} \times V \times \mathfrak{l}=\mathfrak{g},
$$

where $D_{V} \in \operatorname{End}_{\mathfrak{l}}(V)$ is diagonalizable. Since $\mathfrak{g}$ is admissible, there exists $f \in \mathfrak{z}^{\star}$ such that $(V, f \circ \beta)$ is a symplectic l-module of convex type. Hence, every $D_{V}$-eigenspace $V_{\lambda}\left(D_{V}\right) \subseteq V$ defines a symplectic l-module $\left(V_{\lambda}\left(D_{V}\right), f \circ \beta\right)$ of convex type. In particular we have $\beta\left(V_{\lambda}\left(D_{V}\right), V_{\lambda}\left(D_{V}\right)\right) \neq\{0\}$ and, since $D$ is a derivation,

$$
\{0\} \neq \beta\left(V_{\lambda}\left(D_{V}\right), V_{\lambda}\left(D_{V}\right)\right)=\left[V_{\lambda}\left(D_{V}\right), V_{\lambda}\left(D_{V}\right)\right] \subset \mathfrak{z} 2 \lambda\left(D_{\mathfrak{z}}\right) .
$$

Since $\operatorname{spec}(D) \subset\{-1,0,1\}$, this implies $\lambda=0$ and therefore $D_{V}=0$. As $\mathfrak{z}(\mathfrak{g})=$ $[V, V]$, it follows that $D=0$.

Let $\mathfrak{g}=\mathfrak{g}(\mathfrak{l}, V, \mathfrak{z}, \beta)$ be admissible. For $h \in \mathfrak{l}$, we recall the subspaces

$$
V_{h}=[h, V] \text { and } V_{h, 0}=\{v \in V: h . v=0\}=V_{h}^{\perp_{\beta}}
$$

(cf. Lemma 3.1). 
Lemma 4.15 Let $\mathfrak{g}=\mathfrak{g}(\mathfrak{l}, V, \mathfrak{z}, \beta)$ be admissible with $\mathfrak{z} \subseteq[V, V]$ and $h \in[\mathfrak{l}, \mathfrak{l}]$ an Euler element. Then there exists a non-zero Euler derivation $D$ on $\mathfrak{g}$ with $D(\mathfrak{l}) \subseteq \mathfrak{l}$ and $\left.D\right|_{\mathfrak{l}}=\operatorname{ad}_{\mathfrak{l}} h$ if and only if there exists an $\mathfrak{l}$-module decomposition $V_{h}=V_{h}^{+} \oplus V_{h}^{-}$such that the sum of the three subspaces $\left[V_{h}^{+}, V_{h}^{+}\right],\left[V_{h}^{-}, V_{h}^{-}\right]$, and $\left[V_{h}^{+}, V_{h}^{-}\right]+\left[V_{h, 0}, V_{h, 0}\right]$ of $\mathfrak{z}$ is direct.

Proof If $D$ exists, then the invariance of $l$ implies $D(V) \subseteq V$ (Remark 4.11) Therefore $D$ is of the form

$$
D(z, v, y):=\left(D_{\mathfrak{z}} z,[h, v]+D_{V} v,[h, y]\right) \quad \text { for } \quad(z, v, y) \in \mathfrak{z} \times V \times \mathfrak{l}
$$

where $D_{V} \in \operatorname{End}_{\mathfrak{l}}(V)$. Further, the Classification Theorem [17, Thm. 3.14] implies that $\operatorname{ker}\left(D_{V}\right)=V_{h, 0}$, and that $2 h$ acts as an involution on

$$
V_{h}=V_{1 / 2}\left(D_{V}\right)+V_{-1 / 2}\left(D_{V}\right)
$$

We set $V_{h}^{ \pm}:=V_{ \pm 1 / 2}\left(D_{V}\right)$. Then the assertion follows from

$$
\left[V_{h, 0}, V_{h, 0}\right]+\left[V_{h}^{+}, V_{h}^{-}\right]=\mathfrak{z}_{0}\left(D_{\mathfrak{z}}\right) \text { and }\left[V_{h}^{ \pm}, V_{h}^{ \pm}\right]=\mathfrak{z} \pm 1\left(D_{\mathfrak{z}}\right)
$$

Conversely, suppose that the sum of $\left[V_{h}^{+}, V_{h}^{+}\right],\left[V_{h}^{-}, V_{h}^{-}\right]$and $\left[V_{h}^{+}, V_{h}^{-}\right]+$ $\left[V_{h, 0}, V_{h, 0}\right]$ is direct. Since $h$ is an Euler element, the subalgebra $\mathfrak{s}_{h} \subseteq \mathfrak{l}$ generated by $\mathfrak{l}_{ \pm 1}(h)$ is a semisimple ideal of $\mathfrak{l}$ which is a direct sum of hermitian simple ideals of tube type [9, Prop. 3.11(b)]. By Lemma 4.6, the element $2 h$ acts on $V_{\text {eff, } \mathfrak{s}_{h}}=V_{h}$ as an involution. We define

$$
D_{V}:=\left(\frac{1}{2} \mathrm{id}_{V_{h}^{+}}\right) \oplus\left(-\frac{1}{2} \mathrm{id}_{V_{h}^{-}}\right) \oplus 0 \cdot \mathrm{id}_{V_{h, 0}} .
$$

Then $D_{V}$ commutes with the action of $\mathfrak{l}$ on $V$ because $V_{h}^{ \pm}$and $V_{h, 0}$ are l-invariant $\left(\mathfrak{l}=\mathfrak{s}_{h} \oplus \mathfrak{z}_{\mathfrak{l}}\left(\mathfrak{s}_{h}\right)\right)$. Our assumption now implies that we can define an endomorphism $D_{\mathfrak{z}} \in \operatorname{End}(\mathfrak{z})$ by

$$
\mathfrak{z}_{ \pm 1}\left(D_{\mathfrak{z}}\right)=\left[V_{h}^{ \pm}, V_{h}^{ \pm}\right] \quad \text { and } \quad \mathfrak{z}_{0}\left(D_{\mathfrak{z}}\right)=\left[V_{h, 0}, V_{h, 0}\right]+\left[V_{h}^{+}, V_{h}^{-}\right]
$$

Then (4.5) defines an Euler derivation of $\mathfrak{g}$ with $D(\mathfrak{l}) \subseteq \mathfrak{l}$ and $\left.D\right|_{\mathfrak{l}}=$ ad $h$ (cf. [17, Thm. 3.14]).

Remark 4.16 (a) To see the subalgebra $\mathfrak{g}_{D} \subseteq \mathfrak{g}$ generated by $\mathfrak{g}_{ \pm 1}(D)$ in the context of the preceding theorem, we first observe that

$$
\mathfrak{g}_{0}(D)=\mathfrak{z}_{0}\left(D_{\mathfrak{z}}\right) \oplus V_{h,-1 / 2}^{+}(h) \oplus V_{h, 1 / 2}^{-}(h) \oplus V_{h, 0} \oplus \mathfrak{l}_{0}(h) .
$$

For the ideal $\mathfrak{s}_{h} \unlhd \mathfrak{l}$ generated by $h$, we have $\mathfrak{g}_{D} \cap \mathfrak{l}=\mathfrak{s}_{h}$. Next we observe that

$$
\left[\mathfrak{l}_{ \pm 1}(h), V_{h, \mp 1 / 2}^{+}(h)\right]=V_{h, \pm 1 / 2}^{+}(h) .
$$


If $\mathfrak{g}=\mathfrak{g}_{D}$, then we must have $\mathfrak{l}=\mathfrak{s}_{h}$, and then $V=[\mathfrak{l}, V]$ implies $V_{h, 0}=\{0\}$. Conversely, $\mathfrak{l}=\mathfrak{s}_{h}$ and $V_{h, 0}=\{0\}$ imply $\mathfrak{g}=\mathfrak{g}_{D}$ because in this case $\mathfrak{z}_{0}\left(D_{\mathfrak{z}}\right)=$ $\left[V_{h}^{+}, V_{h}^{-}\right]$.

(b) If $\mathfrak{l}=\mathfrak{s}_{h}$ and, as a consequence, $V_{h, 0}=\{0\}$, then we may put $V_{h}^{+}:=V$, which leads to $D_{V}=\frac{1}{2} \mathrm{id}_{V}$ and $D_{\mathfrak{z}}=\mathrm{id}_{\mathfrak{z}}$, so that $D_{\mathfrak{z}} \oplus D_{V}=D_{\text {can }}$. Thus $D=D_{\text {can }}+$ ad $h$ is an Euler derivation of $\mathfrak{g}$ with $\mathfrak{g}_{0}(D)=\left[\mathfrak{g}_{1}(D), \mathfrak{g}_{-1}(D)\right]$.

Theorem 4.17 (Existence of Euler affine pairs) Let $\mathfrak{g}=\mathfrak{g}(\mathfrak{l}, V, \mathfrak{z}, \beta)$ be an admissible Lie algebra with $\mathfrak{z}=\mathfrak{z}(\mathfrak{g}) \subseteq[\mathfrak{g}, \mathfrak{g}]$ and let $x=x_{\mathfrak{z}}+x_{V}+x_{\mathfrak{s}} \in \mathfrak{g}$ be a nilpotent element with $C_{x}$ pointed. Then there exists an Euler derivation $D \in \operatorname{der}(\mathfrak{g})$ with $D x=x$ and $D(\mathfrak{l}) \subseteq \mathfrak{l}$ if and only if there exists an Euler element $h$ of $\mathfrak{l}$ and a decomposition $V_{h}=\bar{V}_{h}^{+} \oplus V_{h}^{-}$into l-submodules such that

(a) $\left[h, x_{\mathfrak{s}}\right]=x_{\mathfrak{s}}$,

(b) $x_{\mathfrak{z}} \in\left[V_{h}^{+}, V_{h}^{+}\right], x_{V} \in V_{h}^{+}$, and

(c) The sum of the three subspaces $\left[V_{h}^{+}, V_{h}^{+}\right],\left[V_{h}^{-}, V_{h}^{-}\right]$, and $\left[V_{h}^{+}, V_{h}^{-}\right]+\left[V_{h, 0}, V_{h, 0}\right]$ of $\mathfrak{z}$ is direct.

Proof Suppose first that an Euler derivation $D$ with $D x=x$ and $D(\mathfrak{l}) \subseteq \mathfrak{l}$ exists. Then, by [17, Thm. 3.14] (here we use that $\mathfrak{z}=\mathfrak{z}(\mathfrak{g}) \subseteq[\mathfrak{g}, \mathfrak{g}]$ ), we have $D(\mathfrak{z}(\mathfrak{l}))=\{0\}$ and there exists an Euler element $h \in[\mathfrak{l}, \mathfrak{l}]$ such that

$$
D(z, v, y):=\left(D_{\mathfrak{z}} z,[h, v]+D_{V} v,[h, y]\right) \quad \text { for } \quad(z, v, y) \in \mathfrak{z} \times V \times \mathfrak{l} .
$$

By Lemma 4.15 and its proof, the l-submodules $V_{h}^{ \pm}:=V_{ \pm 1 / 2}\left(D_{V}\right)$ satisfy (c). Further, (a) is the l-component of $D x=x$, and the first part of (b) follows from

$$
x_{\mathfrak{z}} \in \mathfrak{z}_{1}\left(D_{\mathfrak{z}}\right)=\left[V_{1 / 2}\left(D_{V}\right), V_{1 / 2}\left(D_{V}\right)\right]=\left[V_{h}^{+}, V_{h}^{+}\right]
$$

(cf. [17, Thm. 3.14]). For the second part, we recall from the proof of the Reduction Theorem 3.2 that $x_{V} \in\left[x_{\mathfrak{s}}, V\right]$. As $\left[x_{\mathfrak{s}}, V_{\lambda}(h)\right] \subseteq V_{\lambda+1}(h)$ by (a), we have

$$
\left[x_{\mathfrak{s}}, V\right]=\left[x_{\mathfrak{s}}, V_{-1 / 2}(h)+V_{0}(h)+V_{1 / 2}(h)\right]=\left[x_{\mathfrak{s}}, V_{-1 / 2}(h)\right] \subseteq V_{1 / 2}(h),
$$

hence in particular $x_{V} \in V_{1 / 2}(h)$. Eventually, $D x=x$ entails $x_{V} \in V_{1 / 2}\left(D_{V}\right)=V_{h}^{+}$.

Conversely, suppose that (a)-(c) hold and construct the Euler derivation as in Lemma 4.15 such that

$$
D_{V}:=\left(\frac{1}{2} \mathrm{id}_{V_{h}^{+}}\right) \oplus\left(-\frac{1}{2} \operatorname{id}_{V_{h}^{-}}\right) \oplus 0 \operatorname{id}_{V_{h, 0}} \quad \text { and } \quad \mathfrak{z} \pm 1\left(D_{\mathfrak{z}}\right)=\left[V_{h}^{ \pm}, V_{h}^{ \pm}\right] .
$$

Then $D_{\mathfrak{z}} x_{\mathfrak{z}}=x_{\mathfrak{z}}$ by the first part of (b), and the second part of (b), combined with $x_{V} \in D_{1 / 2}(h)$ implies that

$$
D x_{V}=D_{V} x_{V}+\left[h, x_{V}\right]=\frac{1}{2} x_{V}+\frac{1}{2} x_{V}=x_{V} .
$$

Thus, $D x=x$ follows from (a). 
Here is an example of a rather small admissible Lie algebra that already displays the complexity of the situation we encounter in Theorem 4.17.

Example 4.18 For the Lie algebra $\mathfrak{l}:=\mathbb{R} z \oplus \mathfrak{s l}_{2}(\mathbb{R}) \cong \mathfrak{g l}_{2}(\mathbb{R})$, we consider the representation $\rho$ on

$$
V:=V_{1} \oplus V_{2} \oplus V_{3}, \quad V_{j} \cong \mathbb{R}^{2}
$$

given by

$$
\rho(z)=0 \oplus 0 \oplus\left(\begin{array}{cc}
0 & 1 \\
-1 & 0
\end{array}\right), \quad \rho(x):=x \oplus x \oplus 0 \quad \text { for } \quad x \in \mathfrak{s l}_{2}(\mathbb{R}) .
$$

We then have 3 invariant alternating forms on $V$, represented by

$$
\beta_{+}(\mathbf{v}, \mathbf{w})=v_{1} w_{2}-v_{2} w_{1}, \quad \beta_{-}(\mathbf{v}, \mathbf{w})=v_{3} w_{4}-v_{4} w_{3},
$$

and

$$
\beta_{0}(\mathbf{v}, \mathbf{w})=v_{1} w_{4}-v_{2} w_{3}+v_{3} w_{2}-v_{4} w_{1}+v_{5} w_{6}-v_{6} w_{5} .
$$

For $x:=\left(\begin{array}{cc}0 & 1 \\ -1 & 0\end{array}\right)$, the corresponding Hamiltonians are

$$
\begin{aligned}
& \beta_{+}(x . \mathbf{v}, \mathbf{v})=v_{1}^{2}+v_{2}^{2}, \quad \beta_{-}(x . \mathbf{v}, \mathbf{v})=v_{3}^{2}+v_{4}^{2}, \quad \text { and } \\
& \beta_{0}(x . \mathbf{v}, \mathbf{v})=v_{1} v_{4}+v_{2} v_{3}+v_{4} v_{2}+v_{3} v_{1} .
\end{aligned}
$$

Further, $\beta_{0}(z . \mathbf{v}, \mathbf{v})=v_{5}^{2}+v_{6}^{2}$. Now $\beta=\left(\beta_{+}, \beta_{-}, \beta_{0}\right)$ defines an invariant alternating form with values in $\mathfrak{z}:=\mathbb{R}^{3}$. For $f(x)=2 x_{1}+2 x_{2}+x_{3}$ and $\omega:=f \circ \beta$, the Hamiltonian function $H_{x}$ is positive definite.

For the Euler element $h:=\frac{1}{2} \operatorname{diag}(1,-1) \in \mathfrak{s l}_{2}(\mathbb{R})$, we have

$$
V_{h}=V_{1}+V_{2} \quad \text { and } \quad V_{h, 0}=V_{3}
$$

We define a derivation $D_{\mathfrak{z}}+D_{V}$ on $\mathfrak{h e i s}(V, \beta)$ by $D_{V}=\operatorname{diag}(1 / 2,-1 / 2,0) \otimes \mathrm{id}_{\mathbb{R}^{2}}$ and $D_{\mathfrak{z}}=\operatorname{diag}(1,-1,0)$. Then

$$
\beta\left(V_{h}, V_{h}\right) \nsubseteq \mathfrak{z}_{1}\left(D_{\mathfrak{z}}\right)+\mathfrak{z}-1\left(D_{\mathfrak{z}}\right)
$$

because $\beta_{0}$ does not vanish on $V_{h} \times V_{h}$.

If $D$ is an Euler derivation of $\mathfrak{g}=\mathfrak{g}(\mathfrak{l}, V, \mathfrak{z}, \beta)$ with $\mathfrak{g}_{0}(D)=\left[\mathfrak{g}_{1}(D), \mathfrak{g}_{-1}(D)\right]$, then it induces on $\mathfrak{l} \cong \mathfrak{g} / \mathfrak{u}$ an Euler derivation with $\mathfrak{l}_{0}(D)=\left[\mathfrak{l}_{1}(D), \mathfrak{l}_{-1}(D)\right]$, and this implies in particular that $\mathfrak{l}$ is semisimple and a direct sum of simple hermitian ideals of tube type [9, Prop. 3.11(b)]. This observation justifies the assumption in our final proposition. 
Proposition 4.19 Let $\mathfrak{g}=\mathfrak{g}(\mathfrak{s}, V, \mathfrak{z}, \beta)$ be an admissible Lie algebra, where $\mathfrak{s}$ is a direct sum of hermitian simple Lie algebras of tube type and $\mathfrak{z}=[V, V]$. Let $x \in \mathfrak{g}$ be a nilpotent element with $C_{x}$ pointed. Then there exists an Euler derivation $D \in \operatorname{der}(\mathfrak{g})$ such that

$$
D x=x \quad \text { and } \quad \mathfrak{g}_{0}(D)=\left[\mathfrak{g}_{1}(D), \mathfrak{g}_{-1}(D)\right]
$$

Proof By Corollary 3.3, we may assume that $x=x_{\mathfrak{z}}+x_{\mathfrak{s}} \in \mathfrak{z}+\mathfrak{s}$. Then $x_{\mathfrak{s}}$ is nilpotent with $C_{x_{\mathfrak{s}}}$ pointed (cf. Corollary 3.4). Hence, there exists an Euler element $h \in \mathfrak{s}$ such that $\left[h, x_{\mathfrak{s}}\right]=x_{\mathfrak{s}}$ and such that $\mathfrak{s}$ is generated by $\mathfrak{s}_{ \pm 1}(h)$ [6, Lemma IV.7]. Now the discussion in Remark 4.16(b) shows that $D:=D_{\text {can }}+$ ad $h$ is an Euler derivation satisfying (4.6).

Acknowledgements The funding has been received form Deutsche Forschungsgemeinschaft with Grant no. Ne 413/10-1.

Funding Open Access funding enabled and organized by Projekt DEAL.

Open Access This article is licensed under a Creative Commons Attribution 4.0 International License, which permits use, sharing, adaptation, distribution and reproduction in any medium or format, as long as you give appropriate credit to the original author(s) and the source, provide a link to the Creative Commons licence, and indicate if changes were made. The images or other third party material in this article are included in the article's Creative Commons licence, unless indicated otherwise in a credit line to the material. If material is not included in the article's Creative Commons licence and your intended use is not permitted by statutory regulation or exceeds the permitted use, you will need to obtain permission directly from the copyright holder. To view a copy of this licence, visit http://creativecommons.org/licenses/by/4.0/.

\section{A: Tools Concerning Convexity}

Lemma A.1 [14, Lemma 2.9] If $\emptyset \neq C \subseteq E$ is an open or closed convex subset, then the following assertions hold:

(i) $\lim (C):=\{x \in E: x+C \subseteq C\}$ is a closed convex cone that coincides with $\lim (\bar{C})$.

(ii) $v \in \lim (C)$ if and only if there exist net $t_{j} c_{j} \rightarrow v$, where $t_{j} \geq 0, t_{j} \rightarrow 0$ and $c_{j} \in C$.

(iii) If $c \in C$ and $d \in E$ satisfy $c+\mathbb{R}_{+} d \subseteq C$, then $d \in \lim (C)$.

(iv) $H(C):=\lim (C) \cap-\lim (C)$ is trivial if and only if $C$ contains no affine lines.

Lemma A.2 Let $V$ be a finite dimensional real vector space and $C \subseteq V$ be a closed convex subset. Then the cone $\operatorname{cone}(C):=\overline{\mathbb{R}_{+} C}$ is pointed if $\lim (C)$ is pointed and $0 \notin C$.

Proof First we show that

$$
\operatorname{cone}(C) \subseteq S:=\mathbb{R}_{+} C \cup \lim (C)
$$

Let $v \in \operatorname{cone}(C)$ and choose $\lambda_{n} \geq 0$ and $c_{n} \in C$ with $\lambda_{n} c_{n} \rightarrow v$. 
As $0 \notin C$, the Hahn-Banach Separation Theorem implies the existence of an $f \in V^{*}$ such that $0<\inf f(C)=: \delta$. Then $\lambda_{n} \delta \leq \lambda_{n} f\left(c_{n}\right) \rightarrow f(v)$ shows that the sequence $\lambda_{n}$ is bounded. We may therefore assume that $\lambda_{n} \rightarrow \lambda \in[0, \infty)$. If $\lambda>0$, then the sequence $c_{n}$ converges to $\lambda^{-1} v$ and $v \in \mathbb{R}_{+} C$. If $\lambda=0$, then $v \in \lim (C)$ (Lemma A.1), and (4.1) follows.

Next we observe that $C+\lim (C) \subseteq C$ implies $\mathbb{R}_{+} C+\lim (C) \subseteq \mathbb{R}_{+} C$. Therefore, $S$ is an additive subsemigroup of $V$ in which $\mathbb{R}_{+} C$ is a semigroup ideal, i.e., $S+\mathbb{R}_{+} C \subseteq$ $\mathbb{R}_{+} C$. Since $0 \notin \mathbb{R}_{+} C$, it follows that

$$
S \cap-S \subseteq \lim (C) \cap-\lim (C)=\{0\} .
$$

This implies that cone $(C) \subseteq S$ is pointed.

Lemma A.3 Let $C \subseteq V$ be a pointed closed convex cone and $x \in V$. Then the following are equivalent:

(a) cone $(x+C)=\overline{\mathbb{R}_{+} x+C}$ is pointed.

(b) $(x+C) \cap-C \subseteq\{0\}$.

(c) $0 \notin x+C$ or $x=0$.

Proof $(\mathrm{a}) \Rightarrow(\mathrm{b})$ : Suppose the $D:=\operatorname{cone}(x+C)$ is pointed. Then $C \subseteq D$ and

$$
(x+C) \cap-C \subseteq D \cap-D=\{0\} .
$$

(b) $\Rightarrow$ (c): That the intersection of $x+C$ and $-C$ is contained in $\{0\}$ happens in two cases. If the intersection is empty, then $0 \in-C$ shows that 0 is not contained in $x+C$. If the intersection is non-empty, then it is $\{0\}$ and thus $x \in-C$, which in turn implies $x=0$.

(c) $\Rightarrow$ (a): If $0 \notin x+C$, then Lemma A.2 implies that cone $(x+C)$ is pointed. If $x=0$, then cone $(x+C)=\operatorname{cone}(C)=C$ is trivially pointed.

\section{B: Tools Concerning Lie Algebras}

Proposition B.1 Let $x$ be an element of the semisimple real Lie algebra $\mathfrak{g}$ and $x=$ $x_{s}+x_{n}$ its Jordan decomposition, where $x_{s}$ is semisimple and $x_{n} \neq 0$ is nilpotent. Then there exists a reductive subalgebra $\mathfrak{m} \subseteq \mathfrak{g}$ such that

$$
\mathfrak{m} \cong \mathfrak{s l}_{2}(\mathbb{R}) \quad \text { if } \quad x_{s}=0
$$

and

$$
\mathfrak{m} \cong \mathfrak{g l}_{2}(\mathbb{R}) \quad \text { with } \quad x_{s} \in \mathfrak{z}(\mathfrak{m}), x_{n} \in[\mathfrak{m}, \mathfrak{m}] \quad \text { if } \quad x_{s} \neq 0
$$

Proof Since the Jordan decomposition and the adjoint orbit of $x$ adapts to the decomposition of $\mathfrak{g}$ into simple ideals, we may w.l.o.g. assume that $\mathfrak{g}$ is simple. 
Let $\mathfrak{q}=\mathfrak{l} \ltimes \mathfrak{u} \subseteq \mathfrak{g}$ denote the Jacobson-Morozov parabolic associated with the nilpotent element $x_{n}$ [6]. Then $x_{s} \in \operatorname{ker}\left(\operatorname{ad} x_{n}\right) \subseteq \mathfrak{q}$ implies that $x_{s} \in \mathfrak{q}$. As $x_{s}$ is semisimple, it is conjugate under the group of inner automorphisms of $\mathfrak{q}$ to an element of $\mathfrak{l} .^{1}$ By the Jacobson-Morozov Theorem [1, Ch. VIII, §11, Prop. 2], $\mathfrak{l}$ contains a semisimple element $h$ with $\left[h, x_{n}\right]=2 x_{n}$ and $h \in\left[x_{n}, \mathfrak{g}\right]$. In terms of this element, we have $\mathfrak{q}=\sum_{n>0} \mathfrak{g}_{n}(h)$ and $\mathfrak{l}=\operatorname{ker}(\operatorname{ad} h)$. We further find a nilpotent element $y \in \mathfrak{g}_{-2}(h)$ such that $\left[x_{n}, y\right]=h$, so that the Lie algebra generated by $x$ and $y$ is isomorphic to $\mathfrak{s l}_{2}(\mathbb{R})$. Replacing $x$ by a suitable conjugate, we have seen above that we may assume that $x_{s} \in \mathfrak{g}_{0}(h)$. We consider the Lie algebra

$$
\mathfrak{m}:=\operatorname{span}\left\{x_{s}, h, x_{n}, y\right\}
$$

If $x_{s}=0$, then $\mathfrak{m} \cong \mathfrak{s l}_{2}(\mathbb{R})$. If $x_{s} \neq 0$, then

$$
0=\left[h, x_{s}\right]=\left[\left[x_{n}, y\right], x_{s}\right]=\left[x_{n},\left[y, x_{s}\right]\right] \quad \text { with } \quad\left[y, x_{s}\right] \in \mathfrak{g}_{-2}(h),
$$

so that the representation theory of $\mathfrak{s l}_{2}(\mathbb{R})$ implies that $\left[y, x_{s}\right]=0$. Therefore $x_{s} \in$ $\mathfrak{z}(\mathfrak{m})$.

Corollary B.2 Let $x$ be an element of the semisimple real Lie algebra $\mathfrak{g}$ and $x=x_{s}+x_{n}$ its Jordan decomposition, where $x_{s}$ is semisimple and $x_{n} \neq 0$ is nilpotent. Then the adjoint orbit $\mathcal{O}_{x}$ of $x$ contains all elements of the form $x_{s}+t x_{n}, t>0$. In particular,

$$
x_{s} \in \operatorname{co}(x) \text { and } x_{n} \in \lim (\operatorname{co}(x))
$$

Proof With Proposition B.1 we find an element $h \in \mathfrak{g}$ with $\left[h, x_{s}\right]=0$ and $\left[h, x_{n}\right]=$ $2 x_{n}$. Then the assertion follows from $e^{t \text { ad } h} x=x_{s}+e^{2 t} x_{n}$ for $t \in \mathbb{R}$.

\section{References}

1. Bourbaki, N.: Groupes et algèbres de Lie, Chap. VII-VIII. Masson, Paris (1990)

2. Brunetti, R., Guido, D., Longo, R.: Modular localization and Wigner particles. Rev. Math. Phys. 14, 759-785 (2002)

3. Harish-Chandra (1956) Representations of semi-simple Lie groups, V, VI. Am. J. Math. 78 (1-41), 564-628

4. Hilgert, J., Neeb, K.-H.: Structure and Geometry of Lie Groups. Springer (2012)

5. Hilgert, J., Hofmann, K.H., Lawson, J.D.: Lie Groups, Convex Cones, and Semigroups. Oxford University Press, Oxford (1989)

6. Hilgert, J., Neeb, K.-H., Ørsted, B.: The geometry of nilpotent orbits of convex type in hermitean Lie algebras. J. Lie Theory 4(2), 185-235 (1994)

7. Hochschild, G.P.: Basic Theory of Algebraic Groups and Lie Algebras, Graduate Texts in Mathematics 75. Springer (1981)

8. Lechner, G.: Algebraic constructive quantum field theory: integrable models and deformation techniques. In: Advances in Algebraic Quantum Field Theory," 397-448, Math. Phys. Stud., Springer, Cham (2015). arXiv:1503.03822 [math.ph]

${ }_{1}^{1}$ Every algebraic subgroup $G \subseteq \mathrm{GL}(V), V$ a finite dimensional real vector space, is a semidirect product $G \cong U \rtimes L$, where $U$ is unipotent and $L$ is reductive. Moreover, for every reductive subgroup $L_{1} \subseteq G$ there exists an element $g \in G$ with $g L_{1} g^{-1} \subseteq L$ [7, Thm. VIII.4.3]. 
9. Morinelli, V., Neeb, K.-H.: Covariant homogeneous nets of standard subspaces. Comm. Math. Phys. 386, 305-358 (2021)

10. Neeb, K.-H., Ólafsson, G.: Antiunitary representations and modular theory. In: Grabowska et al., K. (eds) 50th Sophus Lie Seminar, Banach Center Publications 113, pp. 291-362. arXiv:1704.01336 [math-RT]

11. Neeb, K.-H.: Finite dimensional semigroups of unitary endomorphisms of standard subspaces, Representation theory 25, 300-343 (2021). arXiv:1902.02266 [OA]

12. Neeb, K.-H.: Holomorphy and convexity in Lie theory. Expos. Math. 28 (2000) (de Gruyter Berlin)

13. Neeb, K.-H.: The classification of Lie algebras with invariant cones. J. Lie Theory 4, 139-183 (1994)

14. Neeb, K.-H.: Semibounded representations and invariant cones in infinite dimensional Lie algebras. Confluent. Math. 2(1), 37-134 (2010)

15. Oeh, D.: Classification of 3-graded causal subalgebras of real simple Lie algebras, to appear in Transformation Groups (2021). https://doi.org/10.1007/s00031-020-09635-8. arXiv:2001.03125 [math.RT]

16. Oeh, D.: Geometry and construction of standard subspaces from a representation theoretic perspective, PhD Thesis, FAU Erlangen-Nürnberg (2021)

17. Oeh, D.: Lie wedges of endomorphism semigroups of standard subspaces in admissible Lie algebras. arXiv:2007.13445v1 [math.RT]

18. Spindler, K.: Invariante Kegel in Liealgebren. Mitt. Math. Sem. Giessen 188 (1988)

19. Vinberg, E.B.: Invariant cones and orderings in Lie groups. Funct. Anal. Appl. 14, 1-13 (1980)

20. Warner, G.: Harmonic Analysis on Semisimple Lie Groups I. Springer, Berlin, Heidelberg, New York (1972)

Publisher's Note Springer Nature remains neutral with regard to jurisdictional claims in published maps and institutional affiliations. 